\title{
Improving the prediction accuracy of river inflow using two data pre-processing techniques coupled with data-driven model
}

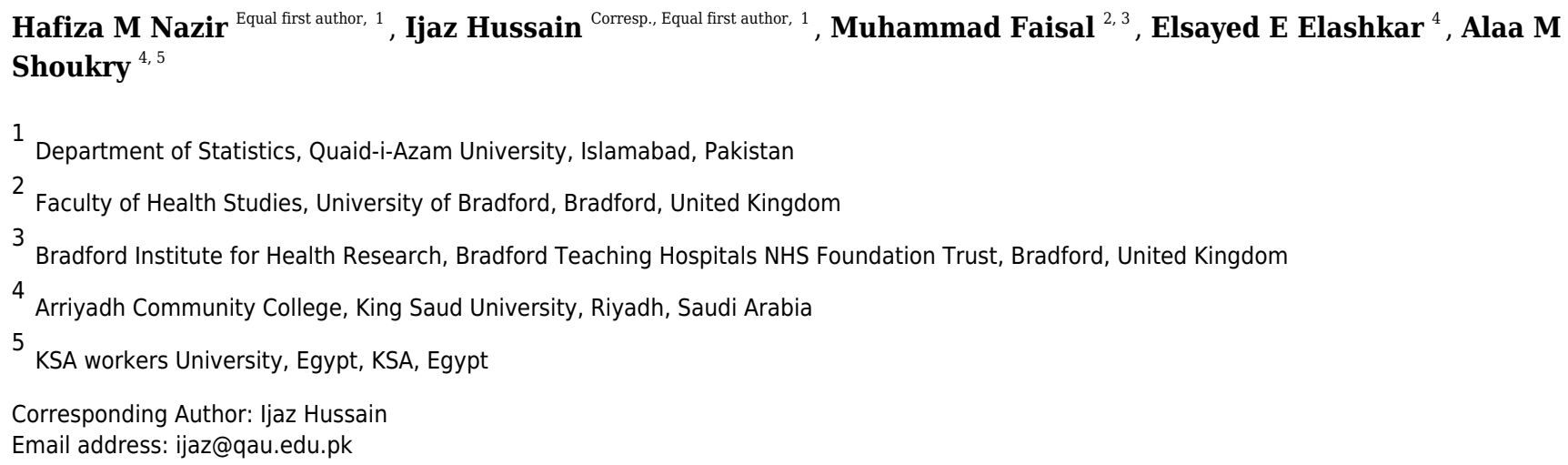

River inflow prediction plays an important role in water resources management and powergenerating systems. But the noises and multi-scale nature of river inflow data adds an extra layer of complexity towards accurate predictive model. To overcome this issue, we proposed a hybrid model, Variational Mode Decomposition (VMD) based on a Singular Spectrum Analysis (SSA) denoising technique. First, SSA has applied to denoise the river inflow data. Second, VMD, a signal processing technique, is employed to decompose the denoised river inflow data into multiple Intrinsic Mode Functions (IMFs), each with a relative frequency scale. Third, Empirical Bayes Threshold (EBT) is applied on non-linear IMF to smooth out. Fourth, predicted models of denoised and decomposed IMFs are established by learning the feature values of the Support Vector Machine (SVM). Finally, the ensemble predicted results are formulated by adding the predicted IMFs. The proposed model is demonstrated using daily river inflow data from four river stations of the Indus River Basin (IRB) system, which is the largest water system in Pakistan. To fully illustrate the superiority of our proposed approach, the SSA-VMD-EBT-SVM hybrid model was compared with SSA-VMD-SVM, VMD-SVM, Empirical Mode Decomposition (EMD) based i.e. EMD-SVM, SSA-EMD-SVM, Ensemble EMD (EEMD) based i.e. EEMD-SVM and SSA-EEMDSVM. We found that our proposed hybrid SSA-EBT-VMD-SVM model outperformed than others based on following performance measures: the Nash-Sutcliffe Efficiency (NSE), Mean Absolute Percentage Error (MAPE) and Root Mean Square Error (RMSE). Therefore, SSA-VMD-EBT-SVM model can be used for water resources management and powergenerating systems using non-linear time series data. 


\title{
Improving the Prediction Accuracy of River Inflow Using Two Data Pre- Processing Techniques Coupled with Data-Driven Model
}

\author{
Hafiza Mamona Nazir ${ }^{1}$, Ijaz Hussain ${ }^{1}$, Muhammad Faisal ${ }^{2,3}$, Elsayed Elsherbini \\ Elashkar ${ }^{4}$, Alaa Mohamd Shoukry,5 \\ ${ }^{1}$ Department of Statistics, Quaid-i-Azam University, Islamabad, Pakistan \\ ${ }^{2}$ Faculty of Health Studies, University of Bradford, Bradford BD7 1DP, UK \\ ${ }^{3}$ Bradford Institute for Health Research, Bradford Teaching Hospitals NHS Foundation Trust, Bradford, UK \\ ${ }^{4}$ Arriyadh Community College, King Saud University Riyadh Saudi Arabia. \\ ${ }^{5}$ KSA workers University, Egypt
}

\section{Abstract}

River inflow prediction plays an important role in water resources management and powergenerating systems. But the noises and multi-scale nature of river inflow data adds an extra layer of complexity towards accurate predictive model. To overcome this issue, we proposed a hybrid model, Variational Mode Decomposition (VMD) based on a Singular Spectrum Analysis (SSA) denoising technique. First, SSA has applied to denoise the river inflow data. Second, VMD, a signal processing technique, is employed to decompose the denoised river inflow data into multiple Intrinsic Mode Functions (IMFs), each with a relative frequency scale. Third, Empirical Bayes Threshold (EBT) is applied on non-linear IMF to smooth out. Fourth, predicted models of denoised and decomposed IMFs are established by learning the feature values of the Support Vector Machine (SVM). Finally, the ensemble predicted results are formulated by adding the predicted IMFs. The proposed model is demonstrated using daily river inflow data from four river stations of the Indus River Basin (IRB) system, which is the largest water system in Pakistan. To fully illustrate the superiority of our proposed approach, the SSA-VMD-EBT-SVM hybrid model was compared with SSA-VMD-SVM, VMD-SVM, Empirical Mode Decomposition (EMD) based i.e. EMD-SVM, SSA-EMD-SVM, Ensemble EMD (EEMD) based i.e. EEMD-SVM and SSA-EEMD-SVM. We found that our proposed hybrid SSA-EBT-VMDSVM model outperforms than others based on following performance measures: the NashSutcliffe Efficiency (NSE), Mean Absolute Percentage Error (MAPE) and Root Mean Square Error (RMSE). Therefore, SSA-VMD-EBT-SVM model can be used for water resources management and power-generating systems using non-linear time series data. 
Keywords: Variational Mode Decomposition, Empirical Mode Decomposition, Ensemble Empirical Mode Decomposition, data-driven models.

Corresponding Author: Ijaz Hussain (ijaz@qau.edu.pk )

\section{Introduction}

Reservoirs are recognized as one of the most powerful tool in integrated water resources management. It considered the major solution in water-related problems like urban and industrial water supply, hydro-power generation, irrigation, flood control and conservation of ecology (ElShafie et al., 2008). However, reservoir system is a challenging problem due to its complexity as reservoirs neither be too empty to operate nor too filled with water to allow capture of flood water (Amnatsan et al., 2018). The optimize reservoir's operation depends on the accuracy of river inflow prediction which is an essential element not only in reservoir operation but also for many hydrological management problems. Accurate prediction results in better decisions such as, flood and drought controls, the supply of drinking water, water resources management and many optimal environmental operations (El-Shafie et al., 2008; Erdal \& Karakurt, 2013; Zhou et al., 2018; Wang et al., 2018; Dehghani et al., 2019). Over the past decades, numerous methods have been developed for accurate river inflow prediction. Literature related to river inflow prediction can be found from these Kisi, 2005; Easey et al., 2006; Londhe \& Charhate, 2010; Adnan et al., a2017; Zaini et al., 2018. These models are broadly classified into three categories: physical-based models, data-driven models, and hybrid models (Chen et al., 2018). All these models have been widely used to predict rivers flow and other hydrologic analyses (Erdal \& Karakurt, 2013; Hao et al., 2017; Chen et al., 2018; Darwen, 2018; Wang et al., 2018). Physicalbased models extract the inherent behaviors of hydrological variables by conceptualizing their physical process and characteristics. However, physical-based models require a large amount of data and detailed mathematical equations, which may raise the issue of estimating huge parameters and the expensive computational costs (Chen et al., 2018). Moreover, due to the unavailability of long hydrological data especially in developing countries, it is difficult to obtain these parameters, which limits the application of these models. Comparative to physical-based models, Data-Driven (DD) models are further classified into Traditional Statistical (TS) and Artificial Intelligence (AI) models to predict linear and non-linear data, respectively. TS models, also called Box and Jenkins methodology (Box \& Jenkins, 1970; Box \& Pierce, 1970, Al-Masudi 
64 et al., 2011) includes the Autoregressive (AR), the Autoregressive Moving Average (ARMA) 65 and the Autoregressive Integrated Moving Average (ARIMA) models are widely applied for predicting river inflow data. Adnan et al. (b2017) used the ARIMA model to predict the streamflow. They took monthly streamflow data and concluded that application of ARIMA can be useful in generating precise prediction. However, the disadvantages of TS models are that the river inflow data must be linear which limits the application of these models (Wang et al., 2018). To overcome these drawbacks, AI models have been introduced which includes Artificial Neural Network (ANN), Multi-Layer Perceptron (MLP), Generalized Regression Neural Network (GRNN), Adaptive Neuro Fuzzy Inference System (ANFIS) (Salih et al., 2019), Multivariate Adaptive Regression (MAR), M5 Model Tree (Yaseen et al., a2016), Support Vector Machine (SVM), Extreme Learning Machine (ELM) (Yaseen et al., b2016), fuzzy logic and Radial Basis Neural Network (RBNN) (Othman \& Naseri, 2011; Yang et al., 2017; Malik \& Kumar, 2018; Mosavi et al., 2018; Kim et al., 2019). These AI techniques have been successively applied in hydrology to accurately predict the river inflow/outflow data (Othman \& Naseri, 2011; Valipour et al. 2013; Shamim et al., 2016; Yang et al., 2017; Malik \& Kumar, 2018; Mosavi et al., 2018). Yaseen et al. (b2016) evaluated the potential of ELM algorithm to validate its superiority over other AI methods and suggested that ELM model outerperform than the other models to predict monthly streamflow. Yaseen et al. (a2016) investigated the usefulness of three types of regression models i.e. least square-SVM, MAR and M5 model tree to forecast the monthly streamflow. Their study indicated that SVM model generally perform superiors than the other models.Among AI techniques, SVM, as the most widely used method, has been considered an effective tool in solving many non-linear mapping relationships to precisely predict rivers flow (Garsole \& Rajurkar, 2015; Adnan et al., 2018; Bafitlhile \& Li, 2019), water level (Behzad et al., 2009) and many other non-linear problems (Wu \& Lin, 2019). However, all these AI models needs to be carefully optimized as hydrological time series data become more and more complex due to rapid climate and other changes. For that purposes, bio-inspired techniques i.e. genetics algorithm, evaluationary programming, differential evolution etc are combined with AI methods to optimize their parameters to enhance its precision (Zheng et al., 2013). But there is a drawback of such bio-inspired based AI methods. First they ignore the multi-scale nature of hydrological data. Second they did not incorporate with noises which is inherited part of hydrological data. So that developing a single model to predict river inflow data is a challenging 
95

96

97

98

99

100

101

102

103

104

105

106

107

108

109

110

111

112

113

114

115

116

117

118

119

120

121

122

123

124

125

task due to its non-stationary, multi-scale and noisiest characteristics (Yang et al., 2016; Yaseen et al., 2017; Yu et al., 2017; Al-Sudani, 2019; Rezaie-Balf et al., a2019; Rezaie-Balf et al., b2019; Rezaie-Balf et al., c2019). Thereby, using the raw river inflow data may not provide useful results, but applying a data pre-processing methods may improve the performance of TS or AI techniques known as hybrid models (Okkan \& Serbes, 2013; Chitsaz et al., 2016; Chen et al., 2018; Wu \& Lin, 2019).

In recent years, hybrid models through data pre-processing techniques have received great attention and commonly applied in non-linear, multi-scale and noisiest time series data such as hydro-meteorology, climatology, finance and economic as powerful alternative modeling tools against alone physical-based or DD models (Chen et al., 2018; Zang et al ., 2018; Rezaie-Balf et al., b2019; Nazir et al., 2019; Wu \& Lin, 2019). Until now, various data pre-processing-based hybrid models have been developed to address these non-linearity issues present in river inflow series. Among all, five main data pre-processing algorithms, i.e., Fourier Analysis, Wavelet Transform (WT) (Daubechies, 1990), and SSA (Golyandina et al., 2001), are combined with TS and AI methods to form a hybrid model. All data pre processing techniques can be used either to decompose non-linear and multi-scale data into the time-frequency domain or to denoise the time series data. Rezaie-Balf et al. (c2019) employed EEMD data pre-processing method to enhance the performance of MAR and M5 Model Tree. They demonstrated that EMD-MAR provides more robust results to predict one-day aheaf river flow. Various studies shows that use of WA have gained popularity in handling multi-scale nature of complex hydrological data by combing with NN and other DD methods. Mouatadid et al (2019) explores the use of WA-Long Shortterm memory network (WA-LSMN) for robust irrigation flow forecasting. Their proposed methodology provides appropriate results rather than the satnd alone LSMN model. Nazir et al. (2019) developed a WA based hybrid model to predict the river inflow data of four stations and shown that their proposed model was better than the simple ARIMA and ANN models. Later, an EBT approach has be developed to enhance the precision of WA (Chipman et al., 1997; Johnstone and Silverman, 2005). In the EBT method, a mixture of priors is selected for the distribution of multi-scale components derived from WA. The posterior median is calculated from selected priors to estimate noise free multi-scale components (To et al., 2009). Moreover, use of EMD (Huang et al., 1998) and EEMD (Wu \& Huang, 2009) with DD models also became 
126 popular to study the non-stationary complex hydrological data (Rezaie-Balf et al., c2019)

127 However, all data pre-processing techniques have some drawbacks with different aspects to 128 decompose the non-linear, multi-scale and noisiest data. The most widely used WA depends

129 heavily on the selection of wavelet basis function (Wang et al., 2018), the application of EMD is

130 limited by its own mathematical mode mixing and sensitivity to denoise property (Nazir et al., 131 2019), and EEMD suffers a strict mathematical theory (Qian et al., 2019; Wu et al., 2019).

132 However, there is a need to develop new hybrid approaches with efficient decomposition 133 methods that predict the non-linear, high irregular and noise-corrupted data with high precision.

134 Several new data pre-processing approaches have been proposed and VMD is commonly used 135 because of its efficient mathematical sound and more precise multi-scale components separation 136 (Ali et al., 2018; Wu \& Lin, 2019; Lei et al., 2019). VMD, as a data decomposition method, has 137 been applied in field of signal processing and wind speed prediction (Liu et a., 2018; Lei et al., 138 2019). Rezaie-Balf et al., (a2019) proposed a new hybrid model comprised on Variational Mode 139 Decomposition based ELM (VMD-ELM) to forecast short term water demand. Their pre140 processing method i.e. VMD provides better results when compared with simple ANN and ELM 141 model. Later, the performance of VMD is enhanced by coupling with EEMD and Random Forest 142 Algorithms (EEMD-VMD-RFA) (Rezaie-Balf et al., c2019).

In this paper, we aimed to develop a novel hybrid model to employ two-phase decomposition based method to efficiently predict the river inflow time series data. Our proposed method comprised on SSA as denoising, VMD as a data decomposition with EBT threshold, and SVM as a prediction method. This work is one of the first attempts known to the authors that used the SSA method as the primary decomposition technique, to enhance the prediction of daily river inflow records with VMD-EBT and SVM

\section{Proposed Methodology}

152 In this paper, a novel hybrid model i.e. SSA-VMD-EBT-SVM is proposed to improve the accuracy of daily river inflow data. The schematic view of proposed methodology is illustrated in Figure 1. The proposed structure is comprised of denoising, decomposition-threshold, prediction and aggregation steps. In denoising stage, SSA is used to denoise the river inflow data (Romero et al., 2015)In the decomposition-threshold stage, VMD is employed to decompose the 
157 denoised daily river inflow series into multiple IMFs (Rezaie-Balf et al., a2019). The high

158 irregular IMF is set as threshold with EBT to remove its sparsity and irregularity (Nazir et al., 159 2019). Further, in the prediction stage, SVM is applied on all IMFs to establish the prediction 160 models and all predicted IMFs are aggregated to get a final prediction (Yaseen et al., a2016). The 161 effectiveness of the proposed hybrid model is evaluated using daily river inflow data from four 162 stations of Indus River Basin (IRB) system, Pakistan (a detail discussion will be in section 3). A 163 brief introduction of SSA, VMD, EBT, and SVM is outlined as follows:

$164 \quad$ 2.1. SSA for denoising

165 For time series analysis, the SSA method is known as a powerful non-parametric method 166 (Golyandina et al., 2001). SSA combines the principals of time series analysis, multivariate 167 statistics, dynamical and signal processing (Suhartono et al., 2018). The reason for using SSA as 168 it is a model-free technique (Romero et al., 2015), which can be applied on any type of data 169 without any assumption. The main function of SSA is to decompose the time series data into a 170 trend, seasonal, oscillations, and aperiodic noises and then reconstruct it after removing aperiodic 171 noises from time series data (Traore, et al., 2017). Unlike other methods of time series analysis, 172 SSA assumes no statistical assumption about noises while performing analysis and investigating 173 its properties (Traore, et al., 2017).

174 2.1.1. Principles of SSA method: The principle of SSA lies in two stages of decomposition and 175 reconstruction, briefly described as follows:

176 Consider a time series data $Y_{1}, Y, \ldots, Y$ of length $N$. The SSA transfer one-dimensional time series 177 data into multi-dimensional $Y_{1}, Y, \ldots, Y_{K}$ where $Y_{i}=\left(y_{1}, y, \ldots, y\right)^{T}$ and $K=N-L+1$. These vectors 178 are grouped into a trajectory matrix as:

$$
Y=\left[\begin{array}{ccc}
y_{1} & \cdots & y_{K} \\
\vdots & \ddots & \vdots \\
y_{L} & \cdots & y_{N}
\end{array}\right]
$$

179 called Hankel matrix whose all the diagonal elements $i+j=$ cons are equal. The only single 180 parameter in this stage is window length $L$ where $2<L<N$ (Traore, 2017). The SSA explores 181 the empirical distribution of pairwise-distances between lagged vectors. The optimality of the 182 SSA method heavily lies on the selection of a window length $L$ as it determines the quality of 183 decomposition (Traore, 2017). To remove noises from original time series values, eigenvalues 184 are calculated from trajectory matrices which can be written as: 


$$
Y=E_{1}, E_{2}, \ldots E_{d}
$$

185 186

187

188

189

190

191

192

193

194

195

196

197

198

199

200

201

202

203

204

205

where $d$ is the number of non-zero eigenvalues in decreasing order $\left(\lambda_{1}, \lambda_{2}, \ldots, \lambda_{d} \geq 0\right)$ of the $L * L$ matrix of $S=Y Y$ and $E_{i}$ is calculated as:

$$
E_{i}=\sqrt{\lambda_{i}} U_{i} V_{i}^{T}
$$

where $i=1,2, \ldots, d, U_{i}$ is eigenvectors and $V_{i}$ is calculated as following:

$$
V_{i}=Y^{T} \cdot \frac{U_{i}}{\sqrt{\lambda_{i}}}
$$

The first few matrices $E_{i}$ to $\mathrm{Y}$ contributed much larger than that of the last few matrices as it is likely that these last matrices represents noises in time series data (Traore, 2017). The next step is to partition the set of indices i.e. $i=1,2, \ldots, d$ into $m$ disjoint subsets i.e. $l_{1}, l_{2}, \ldots l_{m^{*}}$ (Romero et al., 2015). Let one of these partitions $I=i_{1}, \ldots, i_{p}$, then the trajectory matrix of $I$ is defined as $E_{I}$ $=E_{i 1}, \ldots, E_{i p}$. Once the matrices have been calculated for all partitions, then the original time series trajectory matrice is calculated from these partition matrices as $Y=E_{I}=E_{i 1}, \ldots, E_{i p}$. This step is simplified by approximating matrix $Y$ only with first $r$ matrices $Y=E_{1}, \ldots, E_{r}$. The previous step needs a simplification of $r$ parameter appropriately (Romero et al., 2015). An approximated time series then recovered from these subsets of matrices by taking the average of diagonals (Romero et al., 2015; Traore, 2017).

\subsection{VMD as decomposition}

The VMD is a non-recursive signal decomposition estimation method introduced by (Dragomiretskiy \& Zosso, 2014; Rezaie et al., a2019; Rezaie et al., c2019). The VMD adaptively decomposes complicated original non-linear, non-stationary and multi-scale signals into bandlimited IMFs i.e. $u_{k}$ with a specific bandwidth in the spectral domain. To achieve a bandwidth of each IMF, the constrained variational optimization problem is solved as follows (Dragomiretskiy \& Zosso, 2014):

$$
\begin{gathered}
\min _{\left\{u_{k}\right\},\left\{w_{k}\right\}}\left\{\left.\sum_{k}\left\|\partial_{t}\left[\left(\delta(t)+\frac{j}{\pi t}\right) * u_{k}(t)\right] e^{-j w k t}\right\|\right|^{2}\right\} \\
\text { s.t } \sum_{k} u_{k}=f
\end{gathered}
$$


206 where $w_{k}$ is the center frequency of $k t h \operatorname{IMF}, \delta(t)$ is the Dirac function, $t$ is the time script and

$207 k$ is the number of modes. Moreover, $\left(\delta(t)+\frac{j}{\pi t}\right)$ is the Hilbert transformation function which

208 transform $u_{k}$ into an analytical signal to form a one-side frequency. The spectrum of each mode

209 can be shifted to a base-mode with the $e^{-j w k t}$ term. The above constrained problem is converted

210 into unconstrained by making use of a quadratic penalty term i.e. $\alpha$ and Lagrangian multipliers $\lambda$,

211 which is easier to address described as follows:

$$
\begin{gathered}
L\left(\left\{u_{k}\right\},\left\{\omega_{k}\right\}, \lambda\right)=\alpha \sum_{k}\left\|\partial_{t}\left[\left(\delta(t)+\frac{j}{\pi t}\right) * u_{k}(t)\right] e^{-j w k t}\right\| 2+ \\
\left\|f(t)-\sum_{k=1}^{K} u_{k}(t)\right\|_{2}^{2}+\left\langle\lambda(t), f(t)-\sum_{k=1}^{K} u_{k}(t)\right\rangle
\end{gathered}
$$

212 where $\alpha$ denotes balancing parameter. The equation (5) can also be solved by an alternate 213 direction method of multipliers. It is implied that updating $u_{k} \omega_{k}$ and $\lambda_{k}$ in two directions is

214 conducive for realizing the analysis process of VMD, and the solutions of $u_{k} \omega_{k}$ and $\lambda_{k}$ can be 215 calculated as follows:

$$
\begin{aligned}
& u_{k}^{n+1}(\omega)=\frac{\hat{f}(\omega)-\sum_{i \neq k} \hat{u}^{n}(\omega)+\left(\frac{\hat{\lambda}(\omega)}{2}\right)}{1+2 \alpha\left(\omega-\omega_{k}\right)^{2}} \\
& \omega_{k}^{n+1}=\frac{\int_{0}^{\infty} \omega\left|u_{k}^{n+1}(\omega)\right|^{2} d \omega}{\oint_{0}^{\infty}\left|u_{k}^{n+1}(\omega)\right|^{2} d \omega}
\end{aligned}
$$

216 and

$$
\hat{\lambda}_{k}^{n+1}(\omega)=\hat{\lambda}_{k}^{n}(\omega)+\tau\left(\hat{f}(\omega)-\sum_{i \neq k} \hat{u}^{n}(\omega)\right)
$$

217

218 where $\hat{f}(\omega), u_{k}{ }^{n+1}(\omega), u_{k}{ }^{n}(\omega)$ and $\hat{\lambda}(\omega)$ are the Fourier transforms of $f$ and $n$ denotes the 219 number of iterations. The termination condition of VMD is defined as follows:

$$
\frac{\sum_{k}\left\|u_{k}^{n+1}-u_{k}^{n}\right\|_{2}^{2}}{\left\|u_{k}^{n}\right\|_{2}^{2}}<\epsilon
$$

where $\epsilon$ is the tolerance level of the convergence criterion. 
221 From VMD, the IMF $u_{k}$ is obtained from the entire decomposition process according to the

222 following steps:

223 1. Set iteration number $n=1$, and initialize parameters for VMD including $u_{k}{ }^{1}, \omega^{1}$ and

$224 \lambda^{1}$.

2. Using the Equation (8-9), calculate $u_{k}^{n+1}(\omega)$ and $\omega_{k}{ }^{n+1}$.

3. After calculating $u_{k}^{n+1}(\omega)$ and $\omega_{k}^{n+1}$, update Lagrangian multiplier using the Equation (9).

4. If the convergence condition of Equations (10) is met, the iteration will be stopped, otherwise $n$ moves to $n+1$, and again return to step 2. Finally, the IMF are obtained.

\subsubsection{EBT as a threshold}

In EBT method, the posterior distribution is derived with the help of prior distribution to remove sparseness and noises from the coefficients derived from wavelets (To et al., 2009; Nazir et al., 2019). In this study, we used this wavelet-based denoising method to remove noises and sparseness of VMD based coefficients. EBT method has level-dependent thresholding approach which deals each IMF according to its own distribution. EBT assumes a mixture of prior distributions for $k t h$ IMF as follows:

$$
p_{k}\left(I M F_{k} \mid \pi_{k}, \theta\right)=\left(1-\pi_{k}\right) \delta_{0}\left(I M F_{k}\right)+\pi_{k} \gamma(\theta)
$$

where $\pi_{k}$ is the probability of non-zero coefficients of $I M F_{k}, \delta_{0}(\theta)$ presents the Dirac delta function of zero part of $I M F_{k}$ and $\gamma(\theta)$ is a density of non-zero part of $I M F_{k}$. The prior distribution should be chosen in such a way that it belongs to a family of distributions whose tails decays at polynomial rates. In this regard, Laplace distribution, exponential distribution and quasi-Cauchy distribution have been employed for non-zero coefficients of IMFs which are used to estimate noises (Nazir et al., 2019). The probabilities and parameters of a mixture of prior distributions are estimated through maximum likelihood estimation. The reason of using a maximum likelihood approach to estimate unknown is that it determine parameters in such a way that appropriately describe the given data (Hossain et al., 2018). After estimating parameters, the posterior median $\tilde{\theta}_{i}\left(I M F_{k}, \pi_{k}\right)$ is calculated from a mixture of prior distribution as follows:

$$
\widetilde{F_{1}}(\mu \mid i m f)=\int_{\mu}^{\infty} f_{1}(\mu \mid i m f) d \mu
$$


249 which is used as an EBT rule for $\tilde{\mu}$ given data (Johnstone \& Silverman, 2005). Simple hard rule 250 is further applied to estimate noise-free coefficients of IMFs (Johnstone \& Silverman, 2005;

251 Nazir et al., 2019).

252

253

254

255

256

257

258

259

260

261

262

263

264

265

266

267

268

269

270

271

272

\subsection{Support vector machine (SVM) as a prediction method}

The SVM is a supervised machine learning method that comprised of statistical learning principles for nonlinear classification, function estimation, and pattern recognition applications (Vapnik, 1998). After introducing loss function, SVM can be used as a time series forecasting as well (Yaseen et al., a2016; Sanghani et al., 2018). The concept behind the SVM is that it maps the complex non-linear high dimensional data into a high feature space through a nonlinear mapping. After mapping data into a high feature space, linear regression is performed by SVM in that feature space. Let we have a training set consists of $N$ sample points, $\left\{x_{i}, y_{i}\right\}_{i}^{N}$, where $x_{i}$ is lagged input vector and $y_{i}$ is the estimated value of a time series data, then the SVM is formulated as follows:

$$
Y=f(x)=w^{T} \phi(x)+b
$$

where $\phi(x)$ is a non-linear transfer function projecting the input data into high dimensional space, $w_{i}$ are the weight vectors and $b_{i}$ is a bias. Estimating the sampled values with the range of allowed precision is considered as the problem of finding the minimum value for $\|w\|$. This can be summarized as convex programming:

$$
\min \left(\frac{\left\|w^{2}\right\|}{2}+C \sum_{i}^{N}\left(\xi+\xi^{*}\right)\right)
$$

subject to

$$
\left\{\begin{array}{c}
f\left(x_{i}\right)-y_{i} \leq \varepsilon+\xi^{*} \\
y_{i}-f\left(x_{i}\right) \leq \epsilon+\xi \\
\xi, \xi^{*} \geq 0
\end{array}\right.
$$

where $C$ is the user-defined penalty coefficient which represents the dispersion between the weights and objective function. The $\xi$ and $\xi^{*}$ termed as the slack variables which describes how much data exceeds from tolerance. The Lagrangian function is further applied that uses regression function to replace weight vector and $\phi(x)$ given in Equation (13) as follows:

$$
f(x)=\sum_{i}\left(\alpha_{i}-\alpha_{i}{ }^{*}\right) K\left(x, x_{i}\right)+b
$$


273 where $\alpha_{i}$ and $\alpha_{i}{ }^{*}$ are the Lagrangian multipliers and $K$ is called the kernel function. The possible 274 tested kernels includes linear, polynomial, Gaussian and sigmoid kernels which are defined 275 respectively as follows:

$$
\begin{gathered}
K\left(x, x_{i}\right)=x \cdot x_{i} \\
K\left(x, x_{i}\right)=\left(\gamma\left(x . x_{i}\right)+r\right)^{d} \\
K\left(x, x_{i}\right)=\exp \left(-\gamma\left|x-x_{i}\right|^{2}\right) \\
K\left(x, x_{i}\right)=\tanh \left(\gamma\left(x . x_{i}\right)+r\right)
\end{gathered}
$$

276 where $\gamma$ is the structural parameter, $d$ is a polynomial degree and $r$ represents the residuals of the 277 system. Different values of , $\gamma$ and penalty parameter $C$ is used in this study. The quadratic 278 structure of the Equation (16) is defined as:

$$
W\left(\alpha_{i}, \alpha_{i}^{*}\right)=\sum_{i} y_{i}\left(\alpha_{i}-\alpha_{i}^{*}\right)-\varepsilon \sum_{i}\left(\alpha_{i}+\alpha_{i}^{*}\right)-\frac{1}{2} \sum_{i} \sum_{j}\left(\alpha_{i}-\alpha_{i}^{*}\right)\left(\alpha_{j}-\alpha_{j}^{*}\right) K\left(x_{i}, x_{j}\right)
$$

279 With the following constraints:

$$
\begin{gathered}
\sum_{i}^{N}\left(\alpha_{i}-\alpha_{i}^{*}\right)=0 \\
0 \leq \alpha_{i} \leq C, i=1,2, \ldots, N \\
0 \leq \alpha_{i}^{*} \leq C, i=1,2, \ldots, N
\end{gathered}
$$

280

281

282

283

284

285

286

287

\section{Evaluation assessment methods:}

We assessed and compared the predction performance of our proposed hybrid model SSA-VMDEBT-SVM with other existing models (EMD-SVM, EEMD-SVM, VMD-SVM, SSA-EMDSVM, SSA-EEMD-SVM, SSA-VMD-SVM) as a benchmark using following four measures: the Nash-Sutcliffe Efficiency (NSE), Mean Square Error (MSE), Root Mean Square Error (RMSE) (Ghorbani et al., 2018) and Mean Absolute Error (MAE) (Yaseen et al., 2018) with following equations respectively;

$$
\begin{array}{r}
N S E=1-\left[\frac{\sum_{t=1}^{N}\left(y_{o t}-y_{p t}\right)^{2}}{\sum_{t=1}^{N}\left(y_{o t}-\bar{y}_{o t}\right)^{2}}\right] \\
M S E=\frac{\sum_{t=1}^{N}\left(y_{o t}-y_{p t}\right)^{2}}{N}
\end{array}
$$




$$
\begin{aligned}
R M S E & =\sqrt{\frac{\sum_{t=1}^{N}\left(y_{o t}-y_{p t}\right)^{2}}{N}} \\
M A E & =\frac{\sum_{t=1}^{N}\left|y_{o t}-y_{p t}\right|}{N}
\end{aligned}
$$

288 where $y_{o t}$ is the observed values, $\bar{y}_{o t}$ is the mean of observed values and $y_{p t}$ is predicted value of model. Moreover, Taylor diagram is used to prepare a visual comprehension with the help of polar plot for the evaluation of modeling results. The Taylor diagram represents the normalization statndard deviation between simulated and observed values with normalized origin and $R^{2}$ are represented as directional angles (Darbandi \& Pourhosseini, 2018). The interpretation of Taylor diagram is that an observed point is shown on graph and the closer the simulated performance measures to the observe point, the better the model performance (Al-Sudani et al., 2019).

\section{Benchmark models for the evaluation of the proposed hybrid model}

The proposed hybrid model i.e. SSA-VMD-EBT-SVM is compared with six benchmark models described as follows:

a. Without denoising: this type of existing models comprised on decomposition and prediction stages only in which VMD and two different data decomposition methods i.e. EMD, and EEMD are chosen which decompose non-linear, non-stationary and multiscale data into multiple IMFs with the different sound of time-frequency components. For prediction, the extracted IMFs through EMD, EEMD, and VMD are predicted with the same prediction method i.e. SVM as used in our proposed hybrid model. Then, the performance of proposed hybrid model i.e. SSA-VMD-EBT-SVM is compared with existing benchmark models i.e. EMD-SVM (Yu et al., 2017) and EEMD-SVM (RezaieBalf et al. b2019) and VMD-SVM (Wu \& Lin, 2019).

b. With denoising: these models use denoising-decomposition and prediction stages to predict river inflow data. For denoising, SSA is selected with the same decomposition and prediction stages as described in (a). Then, the performance of the proposed hybrid model i.e. SSA-VMD-EBT-SVM is compared with existing benchmark models i.e. SSAEMD-SVM, SSA-EEMD-SVM, and SSA-VMD-SVM.

\section{Case study and experimental design}

The largest water system in Pakistan i.e. IRB is considered for application of proposed architecture as the IRB is Pakistan's largest source of power generation, irrigation, and insensible water resource system. Data from its four major rivers are analyzed i.e. the River Indus, the 
318 River Jhelum, the River Chenab, and the River Kabul which contributed significantly in the 319 water system of IRB. The reason of selecting these tributaries is that they are facing frequent 320 river flooding each year due to heavy monsoon rain and melting snow or glacier in Pakistan, 321 glacier-covered $13,680 \mathrm{~km}^{2}$ area which is estimated $13 \%$ of the mountainous areas of Upper 322 Indus Basin (UIB). Melted water from these 13\% areas adds a significant contribution of water 323 in these rivers. Therefore, it is appropriate to use rivers data of IRB as a representative case 324 study for evaluation of the proposed model.

\subsection{Data}

326 The daily river inflow dataset used in this study is comprised on $1^{\text {st }}$ January to $31^{\text {st }}$ March for the 327 period of 2015-2019. To the application of proposed objective, the daily inflow of Indus River at 328 Tarbela with its two principal left and one right bank tributaries: Jhelum River at Mangla, 329 Chenab River at Marala and Kabul River at Nowshera respectively are selected. The daily inflow 330 data is measured in $1000 \mathrm{ft} / \mathrm{s}$ which was acquired from the site of Pakistan Water and Power 331 Development Authority (WAPDA).

332

\section{Results}

Results of the proposed hybrid model i.e. SSA-VMD-EBT-SVM is defined in stages as follows:

Denoise-stage results: first, Augmented Dickey-Fuller (ADF) (Said \& Dickey, 1985) test is applied on river inflow data of all selected case studies to confirm the non-stationarity. For all case studies, results of the ADF test showed that river inflow data is non-stationary in nature with p-values listed in Table 1. Then the original non-stationary data is processed with SSA to improve the quality of river inflow data by reducing the noises. In processing SSA, window length and number of group i.e. $L$ and $m$ parameter must be determined respectively. Here, different values of $L$ are tested and the optimal value i.e. 90 is selected that gives the lowest error rate of actual and denoised series. The value of $m$ is selected according to the eigenvalues of each river inflow.

344 The eigenvalues of four selected four rivers of IRB system are shown in Figure 2 for Indus and Jhelum river inflow, which shows that the values of components of 30, 25, 30 and 20 for Indus, Jhelum, Chenab and Kabul river inflow respectively are clearly larger than those of the remaining components. The denoised river inflow data is reconstructed by using the selected 
349 and standard deviation of original and denoised river inflow data is listed in Table 2 where it can

350 be observed that mean value remains the same while the standard deviation of denoised river 351 inflow is reduced through processing.

352

353 Decomposition-stage results: after the original river inflow data is processed with SSA, the 354 denoised data is decomposed into linear and non-linear time-scale oscillations called IMFs by

355

356

357

358

359

360

361

362

363

364

365

366

367

368

369

370

371

372

373

374

375

376

377

378

379 VMD. The number of IMFs i.e. $K$ must be selected in advance in order to proceed with any decomposition method. Here, $K=6$ is selected as the remaining IMFs tend to be similar when $K>6$. All river inflow data is decomposed into six IMFs. The decomposition results of Indus river inflow is shown in Figure 4. For comparison, the same river inflow data is also decomposed with EMD and EEMD as shown in Figure 4 and 5 respectively for Indus river inflow. It can be seen from Figure 3, 4 and 5 that the IMFs extracted through VMD are smoother than the other decomposition methods i.e. EMD and EEMD. However, due to the high oscillations of sixth IMF, extracted through VMD, EBT is applied to denoise IMF. The EBT effectively separate the clear and noisy coefficients of noise dominant IMF by a mixture of prior distributions as defined in Eq. (12) and preserve valid information as much as possible. First, to get normal distribution, the scaled transformation is applied so that each IMF follows $N\left(\delta_{i}, 1\right)$. According to the nature of the sixth IMF as depicted in Figure 4, the first two IMFs of Figure 5 and the first three IMFs of Figure 6 it is known that most of the coefficients in all noisiest IMFs are zero and few are nonzero out of which fewer coefficients are either very low or very high in magnitude. By inspecting both zero and non-zero coefficients of IMFs, a mixture of an atom of probability at zero and different distributions are considered for non-zero part coefficients of IMF (Johnstone \& Silverman, 2005). Laplace distribution is chosen as a prior distribution out of Exponential and Cauchy distribution for $\delta_{i}$. Finally, the valid information of IMFs are preserved with posterior median threshold estimator which is calculated through Eq. (13).

Prediction results: finally, the denoised and decomposed IMFs of the proposed model i.e. SSAVMD-EBT for all selected rivers are predicted through SVM. For that purpose, the daily river inflow data from $1^{\text {st }}$ Jan-2015 to $31^{\text {st }}$ Dec-2018 i.e. 1461 observations are used for training and $1^{\text {st }}$ Jan-2019 to $31^{\text {st }}$ Mar-2019 i.e. 90 observations are used for testing purposes. The SVM is trained by setting different values of $\gamma$ and penalty parameter $C$. The values of $\gamma=10$ and $C=10000$ 
380

381

382

383

384

385

386

387

388

389

390

391

392

393

394

395

396

397

398

399

400

401

402

403

404

405

406

407

408

409

410

are selected. The parameters of SVM are determined using trial and error basis on which error of training and testing is minimized. according to minimum prediction error for all river inflow. After estimation of each IMF, the accuracy of proposed and benchmark models are measured with NSE, MSE, RMSE, MAE, and MAPE. The performance of proposed hybrid model i.e. SSA-VMD-EBT-SVM is compared with 'without denoising model' i.e. EMD-SVM and EEMDSVM and VMD-SVM and 'with denoising models' i.e. SSA-EMD-SVM, SSA-EEMD-SVM, and SSA-VMD-SVM. The training results of proposed model i.e. SSA-VMD-EBT-SVM with comparison to all benchmark models for Indus, Jhelum and Chenab river inflow data are listed in Table 3 and results of Kabul river inflow data is presented in Table 4. Moreover, Taylor diagram, as shown in Figure 7, is used to illustrate the efficiency of proposed model. The graph shows that the proposed model performed very well over other exisiting models. From Table 3 and 4 and Figure 7, it is concluded that the proposed model results fully demonstrate the effectiveness for all four cases studies with minimum NSE, MSE, RMSE, MAE, and MAPE compared to all with and without denoising models. The worst prediction models are those which are handled through without denoising i.e. EMD-SVM and EEMD-SVM. The validation graphs of proposed model i.e. SSA-VMD-EBT-SVM with comparison to with denoising models i.e. SSA-EMD-SVM, SSA-EEMD-SVM and SSA-VMD-SVM and without denoising models i.e. EMD-SVM and EEMD-SVM and VMD-SVM for Indus and Jhelum river inflow are shown in Figure 8. From graph, it can be observed that our proposed model i.e. SSA-VMD-EBT-SVM performed well not only in prediction stage but also in validation. However, the exisiting models perform only good in prediction stage as shown in Figure 8.

\section{Discussion}

In this paper, we proposed a novel hybrid model to efficiently predict the river inflow time series data. Our proposed method comprised of SSA as denoising, VMD as a data decomposition with EBT threshold, and SVM as a prediction method.

In order to understand the applicability of our proposed model i.e. SSA-VMD-EBT-SVM, two different model strategies are adapted (see Table 3 and 4). First, without denoising model strategy is implemented on which the same decomposition method i.e. VMD and two different decomposition methods i.e. EMD and EEMD are used. Moreover, for prediction purpose, SVM which is also adapted in proposed methodology is used here to compare the performance of proposed denoised-decomposed strategy with only 'without denoising models i.e. VMD-SVM 
411 (Wu \& Lin, 2019), EMD-SVM (Yu et al., 2017) and EEMD-SVM (Rezaie-Balf et al., b2019).

412 From Table 3 and 4, it can be seen that the overall performance of without denoising models are 413 poor for all river inflow data with high NSE, MSE, RMSE, MAE and MAPE values. 414 Specifically, EMD-SVM performed worst among all without denoising models due to the fact 415 that EMD suffers from the mode mixing problem and fail to produce noiseless IMFs (Di et al., 416 2014). The proposed model performed well with the lowest NSE, MSE, RMSE, MAE and 417 MAPE values as compared to without denoising models.

418 The second strategy used the concept of denoising and decomposition, here the same method of 419 denoising which is used in proposed methodology is employed with different decomposition 420 methods i.e. SSA-EMD-SVM, SSA-EEMD-SVM and with same decomposition method but 421 without thresholding is adapted i.e. SSA-VMD-SVM. From Table 3 and 4, it is observed that the 422 proposed model i.e. SSA-VMD-EBT-SVM performed well for Indus, Jhelum, and Chenab, but 423 for Kabul river inflow the results of SSA-VMD-EBT-SVM and SSA-VMD-SVM are same as 424 thresholding the IMF did not enhance the prediction performance of SVM. Moreover, SSA425 EEMD-SVM also performs well among existing with and without denoising methods for all case 426 studies. Overall, the proposed SSA-VMD-EBT-SVM model showed a much better agreement 427 between predicted and observed river inflow data which demonstrates the suitability of SSA, 428 VMD, and EBT in pre-processing inputs/output data over other decomposition methods i.e. 429 EMD and EEMD. Thus, it is concluded that the appropriate way of denoising, decomposition 430 and thresholding can effectively enhance the performance of non-linear, non-stationary and 431 multi-scale time series data.

432 By applying proposed simulation models in IRB, it is expected that this will provide new tools 433 for improving inflow prediction over what is possible with the current generation of statistical 434 models as well as help with other land and water management questions. It may also be helpful 435 in setting policies regarding what appropriate methods should be chosen for 'denoising436 decomposition and prediction' and in assessing the effects of climate warming. These modeling 437 efforts are therefore significant both for the scientific issues involved as well as for the practical 438 relevance of the results. 
441 The reliable and accurate prediction of river inflow is essential in order to manage water

442 resources. In this paper, a hybrid prediction model i.e. SSA-VMD-EBT-SVM is proposed and 443 applied for the prediction of daily river inflow data of four rivers of IRB. The original river 444 inflow data is denoised with SSA and decomposed into several linear and non-linear IMFs by 445 using VMD, then EBT is applied on non-linear IMF to remove noises and sparsities. Finally, 446 each IMF is predicted with SVM and the predicted results of IMF component are aggregated as 447 the final prediction results. To compare the performance of the proposed model, the benchmark 448 model with two different decomposition methods i.e. EMD and EEMD methods combined with 449 SSA-based denoising and without denoising is selected. The five performance indicators NSE, 450 MSE, MAE, RMSE, and MAPE are employed to measure the prediction accuracy of proposed 451 SSA-VMD-EBT-SVM, and all other benchmark models. Based on the results, it is observed that 452 the proposed hybrid model i.e. SSA-VMD-EBT-SVM shown the efficient results with minimum 453 errors. In other words, compared with other models, the proposed hybrid model improves 454 prediction accuracy and reduces errors. The results from this research will not only beneficial for 455 sustainable water resource management but also for other non-linear time series data.

456

457

458

459

460

461

462

463

\section{References}

1. Adnan, R. M., Yuan, X., Kisi, O., Adnan, M., \& Mehmood, A. (2018). Stream Flow Forecasting of Poorly Gauged Mountainous Watershed by Least Square Support Vector Machine, Fuzzy Genetic Algorithm and M5 Model Tree Using Climatic Data from Nearby Station. Water resources management, 32(14), 4469-4486.

2. Adnan, R. M., Yuan, X., Kisi, O., \& Yuan, Y. (2a017). Streamflow forecasting using artificial neural network and support vector machine models. American Scientific Research Journal for Engineering, Technology, and Sciences (ASRJETS), 29(1), 286-294.

3. Adnan, R. M., Yuan, X., Kisi, O., \& Curtef, V. (b2017). Application of time series models for streamflow forecasting. Civil Environ Res, 9(3), 56-63.

4. Ali, M., Khan, A., \& Rehman, N. U. (2018). Hybrid multiscale wind speed forecasting based on variational mode decomposition. International Transactions on Electrical Energy Systems, 28(1).

5. Al-Masudi, R. K. (2011). Fitting ARIMA Models for Forecasting to inflow of Dokan Reservoir. Journal of Babylon University, 19(4). 
472 6. Al-Sudani, Z. A., Salih, S. Q., \& Yaseen, Z. M. (2019). Development of multivariate

473

474

475

476

477

478

479

480

481

482

483

484

485

486

487

488

489

490

491

492

493

494

495

496

497

498

499

500

501

502 adaptive regression spline integrated with differential evolution model for streamflow simulation. Journal of Hydrology, 573, 1-12.

7. Amnatsan, S., Yoshikawa, S., \& Kanae, S. (2018). Improved Forecasting of Extreme Monthly Reservoir Inflow Using an Analogue-Based Forecasting Method: A Case Study of the Sirikit Dam in Thailand. Water, 10(11), 1614.

8. Bafitlhile, T. M., \& Li, Z. (2019). Applicability of $\varepsilon$-Support Vector Machine and Artificial Neural Network for Flood Forecasting in Humid, Semi-Humid and Semi-Arid Basins in China. Water, 11(1), 85.

9. Behzad, M., Asghari, K., \& Coppola Jr, E. A. (2009). Comparative study of SVMs and ANNs in aquifer water level prediction. Journal of Computing in Civil Engineering, 24(5), 408-413.

10. Box, G.E.P. \& Jenkins, G. (1970). Time series analysis, forecasting and control. In Time Series Analysis, Forecasting and Control. INSPEC:209917, Holden-Day: San Francisco, CA, USA, 19-553.

11. Box, G.E.P. \& Pierce, D.A. (1970). Distribution of residual autocorrelations in autoregressive-integrated moving time series models. J. Am. Stat. Assoc, 65, 1509-1526.

12. Chen, L., Sun, N., Zhou, C., Zhou, J., Zhou, Y., Zhang, J., \& Zhou, Q. (2018). Flood forecasting based on an improved extreme learning machine model combined with the backtracking search optimization algorithm. Water, 10(10), 1362.

13. Chitsaz, N., Azarnivand, A., \& Araghinejad, S. (2016). Pre-processing of data-driven river flow forecasting models by singular value decomposition (SVD) technique. Hydrological Sciences Journal, 61(12), 2164-2178.

14. Chipman, H. A., Kolaczyk, E. D., \& McCulloch, R. E. (1997). Adaptive Bayesian wavelet shrinkage. Journal of the American Statistical Association, 92(440), 1413-1421.

15. Darbandi, S., \& Pourhosseini, F. A. (2018). River flow simulation using a multilayer perceptron-firefly algorithm model. Applied Water Science, 8(3), 85.

16. Darwen, P. J. (2019). Bayesian model averaging for river flow prediction. Applied Intelligence, 49(1), 103-111.

17. Daubechies, I. (1990). The wavelet transform, time-frequency localization and signal analysis. IEEE transactions on information theory, 36(5), 961-1005. 
503 18. Dehghani, M., Riahi-Madvar, H., Hooshyaripor, F., Mosavi, A., Shamshirband, S.,

504 Zavadskas, E. K., \& Chau, K. W. (2019). Prediction of hydropower generation using grey 505 wolf optimization adaptive neuro-fuzzy inference system. Energies, 12(2), 289.

506 19. Di, C., Yang, X., \& Wang, X. (2014). A four-stage hybrid model for hydrological time series 507 forecasting. PloS one, 9(8), e104663.

508 20. Dragomiretskiy, K., \& Zosso, D. (2014). Variational mode decomposition. IEEE 509 transactions on signal processing, 62(3), 531-544.

510 21. Easey, J., Prudhomme, C., \& Hannah, D. M. (2006). Seasonal forecasting of river flows: a 511 review of the state-of-the-art. IAHS publication, 308, 158.

512 22. El-Shafie, A., Noureldin, A. E., Taha, M. R., \& Basri, H. (2008). Neural network model for 513 Nile river inflow forecasting based on correlation analysis of historical inflow data. $J$ Appl $514 \quad$ Sci, 8(24), 4487-4499.

515 23. Erdal, H. I., \& Karakurt, O. (2013). Advancing monthly streamflow prediction accuracy of 516 CART models using ensemble learning paradigms. Journal of Hydrology, 477, 119-128.

517 24. Fathian, F., Mehdizadeh, S., Sales, A. K., \& Safari, M. J. S. (2019). Hybrid models to 518 improve the monthly river flow prediction: Integrating artificial intelligence and non-linear 519 time series models. Journal of Hydrology.

520 25. Garsole, P., \& Rajurkar, M. (2015). Streamflow forecasting by using support vector 521 regression. In Proc., 20th Int. Conf. of Hydraulics, Water Resources and River Engineering.

522 26. Ghorbani, M. A., Khatibi, R., Karimi, V., Yaseen, Z. M., \& Zounemat-Kermani, M. (2018).

523 Learning from multiple models using artificial intelligence to improve model prediction 524 accuracies: application to river flows. Water resources management, 32(13), 4201-4215.

525

526 27. Golyandina, N., Nekrutkin, V., \& Zhigljavsky, A. A. (2001). Analysis of time series 527 structure: SSA and related techniques. Chapman and Hall/CRC.

528 28. Hao, Z., Hao, F., Singh, V. P., Ouyang, W., \& Cheng, H. (2017). An integrated package for 529 drought monitoring, prediction and analysis to aid drought modeling and assessment. 530 Environmental modelling \& software, 91, 199-209.

29. Hossain MM, Kozubowski TJ, Podgórski K. 2018. A novel weighted likelihood estimation with empirical Bayes flavor. Communications in Statistics-Simulation and Computation, $47(2), 392-412$. 
534 30. Huang, N. E., Shen, Z., Long, S. R., Wu, M. C., Shih, H. H., Zheng, Q., ... \& Liu, H. H. 535 (1998). The empirical mode decomposition and the Hilbert spectrum for nonlinear and non536 stationary time series analysis. Proceedings of the Royal Society of London. Series A: 537 Mathematical, Physical and Engineering Sciences, 454(1971), 903-995.

538 31. Johnstone, I. M., \& Silverman, B. W. (2005). Empirical Bayes selection of wavelet 539 thresholds. Annals of Statistics, 1700-1752.

540 32. Kim, T., Shin, J. Y., Kim, H., Kim, S., \& Heo, J. H. (2019). The Use of Large-Scale Climate 541 Indices in Monthly Reservoir Inflow Forecasting and Its Application on Time Series and $542 \quad$ Artificial Intelligence Models. Water, 11(2), 374.

543 33. Kisi, O. (2005). Daily river flow forecasting using artificial neural networks and auto544 regressive models. Turkish Journal of Engineering and Environmental Sciences, 29(1), 9-20.

545 34. Lei, Z., Su, W., \& Hu, Q. (2019). Multimode Decomposition and Wavelet Threshold 546 Denoising of Mold Level Based on Mutual Information Entropy. Entropy, 21(2), 202.

547 35. Liu, H., Mi, X., \& Li, Y. (2018). Smart multi-step deep learning model for wind speed 548 forecasting based on variational mode decomposition, singular spectrum analysis, LSTM 549 network and ELM. Energy Conversion and Management, 159, 54-64.

550 36. Londhe, S., \& Charhate, S. (2010). Comparison of data-driven modelling techniques for river 551 flow forecasting. Hydrological Sciences Journal-Journal des Sciences Hydrologiques, 55(7), 552 1163-1174.

553 37. Malik, A., \& Kumar, A. (2018). Comparison of soft-computing and statistical techniques in 554 simulating daily river flow: a case study in India. Journal of Soil and Water Conservation, $555 \quad 17(2), 192-199$.

556 38. Mosavi, A., Ozturk, P., \& Chau, K. W. (2018). Flood prediction using machine learning 557 models: Literature review. Water, 10(11), 1536.

558 39. Mouatadid, S., Adamowski, J. F., Tiwari, M. K., \& Quilty, J. M. (2019). Coupling the 559 maximum overlap discrete wavelet transform and long short-term memory networks for 560 irrigation flow forecasting. Agricultural Water Management, 219, 72-85.

561 40. Nazir, H. M., Hussain, I., Faisal, M., Shoukry, A. M., Gani, S., \& Ahmad, I. (2019). 562 Development of Multidecomposition Hybrid Model for Hydrological Time Series Analysis. 563 Complexity. 
564 41. Okkan, U., \& Serbes, Z. A. (2013). The combined use of wavelet transform and black box 565 models in reservoir inflow modeling. Journal of Hydrology and Hydromechanics, 61(2), $566 \quad 112-119$

567

568

569

570

571

572

573

574

575

576

577

578

579

580

581

582

583

584

585

586

587

588

589

590

591

592

42. Othman, F., \& Naseri, M. (2011). Reservoir inflow forecasting using artificial neural network. International journal of physical sciences, 6(3), 434-440.

43. Qian, Z., Pei, Y., Zareipour, H., \& Chen, N. (2019). A review and discussion of decomposition-based hybrid models for wind energy forecasting applications. Applied energy, 235, 939-953.

44. Rezaie-Balf, M., Fani Nowbandegani, S., Samadi, S. Z., Fallah, H., \& Alaghmand, S. (a2019). An Ensemble Decomposition-Based Artificial Intelligence Approach for Daily Streamflow Prediction. Water, 11(4), 709. Rezaie-Balf, M., Kisi, O., \& Chua, L. H. (b2019). Application of ensemble empirical mode decomposition based on machine learning methodologies in forecasting monthly pan evaporation. Hydrology Research, 50(2), 498-516.

45. Rezaie-Balf, M., Kim, S., Fallah, H., \& Alaghmand, S. (c2019). Daily river flow forecasting using ensemble empirical mode decomposition based heuristic regression models: Application on the perennial rivers in Iran and South Korea. Journal of Hydrology, 572, 470485.

46. Romero, F., Alonso, F. J., Cubero, J., \& Galán-Marín, G. (2015). An automatic SSA-based de-noising and smoothing technique for surface electromyography signals. Biomedical Signal Processing and Control, 18, 317-324.

47. Said, S. E., \& Dickey, D. A. (1985). Hypothesis testing in ARIMA (p, 1, q) models. Journal of the American Statistical Association, 80(390), 369-374.

48. Salih, S. Q., Allawi, M. F., Yousif, A. A., Armanuos, A. M., Saggi, M. K., Ali, M., ... \& Chau, K. W. (2019). Viability of the advanced adaptive neuro-fuzzy inference system model on reservoir evaporation process simulation: case study of Nasser Lake in Egypt. Engineering Applications of Computational Fluid Mechanics, 13(1), 878-891.

49. Sanghani, A., Bhatt, N., \& Chauhan, N. C. (2018). A Novel Hybrid Method for Time Series Forecasting Using Soft Computing Approach. In International Conference on ISMAC in Computational Vision and Bio-Engineering, 1123-1134. 
593 50. Shamim, M. A., Hassan, M., Ahmad, S., \& Zeeshan, M. (2016). A comparison of artificial 594 neural networks (ANN) and local linear regression (LLR) techniques for predicting monthly 595 reservoir levels. KSCE Journal of Civil Engineering, 20(2), 971-977.

596

597

51. Suhartono, S., Isnawati, S., Salehah, N. A., Prastyo, D. D., Kuswanto, H., \& Lee, M. H. (2018). Hybrid SSA-TSR-ARIMA for water demand forecasting. International Journal of Advances in Intelligent Informatics, 4(3), 238-250.

52. To, A. C., Moore, J. R., \& Glaser, S. D. (2009). Wavelet denoising techniques with 600 applications to experimental geophysical data. Signal Processing, 89(2), 144-160.

53. Traore, O. I., Pantera, L., Favretto-Cristini, N., Cristini, P., Viguier-Pla, S., \& Vieu, P.

602 (2017). Structure analysis and denoising using Singular Spectrum Analysis: Application to acoustic emission signals from nuclear safety experiments. Measurement, 104, 78-88.

54. Valipour, M., Banihabib, M. E., \& Behbahani, S. M. R. (2013). Comparison of the ARMA, ARIMA, and the autoregressive artificial neural network models in forecasting the monthly inflow of Dez dam reservoir. Journal of hydrology, 476, 433-441.

55. Vapnik, V. (1998). The support vector method of function estimation. In Nonlinear Modeling (pp. 55-85). Springer, Boston, MA.

56. Wang, Z. Y., Qiu, J., \& Li, F. F. (2018). Hybrid models combining EMD/EEMD and ARIMA for Long-term streamflow forecasting. Water, 10(7), 853.

57. Wang, L., Li, X., Ma, C., \& Bai, Y. (2019). Improving the prediction accuracy of monthly streamflow using a data-driven model based on a double-processing strategy. Journal of Hydrology, 573, 733-745.

58. Wu, Q., \& Lin, H. (2019). Short-Term Wind Speed Forecasting Based on Hybrid Variational Mode Decomposition and Least Squares Support Vector Machine Optimized by Bat Algorithm Model. Sustainability, 11(3), 652.

59. Wu, Z., \& Huang, N. E. (2009). Ensemble empirical mode decomposition: a noise-assisted data analysis method. Advances in adaptive data analysis, 1(01), 1-41.

60. Yaseen, Z. M., Kisi, O., \& Demir, V. (a2016). Enhancing long-term streamflow forecasting and predicting using periodicity data component: application of artificial intelligence. Water resources management, 30(12), 4125-4151. 
622 61. Yaseen, Z. M., Jaafar, O., Deo, R. C., Kisi, O., Adamowski, J., Quilty, J., \& El-Shafie, A. 623 (b2016). Stream-flow forecasting using extreme learning machines: A case study in a semi624 arid region in Iraq. Journal of Hydrology, 542, 603-614.

625 62. Yaseen, Z. M., Ebtehaj, I., Bonakdari, H., Deo, R. C., Mehr, A. D., Mohtar, W. H. M. W., ... $626 \&$ Singh, V. P. (2017). Novel approach for streamflow forecasting using a hybrid ANFIS627 FFA model. Journal of Hydrology, 554, 263-276.

628 63. Yaseen, Z. M., Fu, M., Wang, C., Mohtar, W. H. M. W., Deo, R. C., \& El-Shafie, A. (2018). 629 Application of the hybrid artificial neural network coupled with rolling mechanism and grey 630 model algorithms for streamflow forecasting over multiple time horizons. Water resources 631 management, 32(5), 1883-1899.

632 64. Yang, T., Asanjan, A. A., Welles, E., Gao, X., Sorooshian, S., \& Liu, X. (2017). Developing 633 reservoir monthly inflow forecasts using artificial intelligence and climate phenomenon information. Water Resources Research, 53(4), 2786-2812.

65. Yang, M., Sang, Y. F., Liu, C., \& Wang, Z. (2016). Discussion on the choice of decomposition level for wavelet based hydrological time series modeling. Water, 8(5), 197.

66. Yaseen, Z. M., Jaafar, O., Deo, R. C., Kisi, O., Adamowski, J., Quilty, J., \& El-Shafie, A. (2016). Stream-flow forecasting using extreme learning machines: A case study in a semiarid region in Iraq. Journal of Hydrology, 542, 603-614.

67. Yu, Y., Wang, P., Wang, C., Qian, J., \& Hou, J. (2017). Combined monthly inflow forecasting and multiobjective ecological reservoir operations model: Case study of the Three Gorges Reservoir. Journal of Water Resources Planning and Management, 143(8), 05017004.

68. Zaini, N., Malek, M. A., Yusoff, M., Mardi, N. H., \& Norhisham, S. (2018). Daily River Flow Forecasting with Hybrid Support Vector Machine-Particle Swarm Optimization. In IOP Conference Series: Earth and Environmental Science (Vol. 140, No. 1, p. 012035). IOP Publishing.

69. Zhou, J., Peng, T., Zhang, C., \& Sun, N. (2018). Data pre-analysis and ensemble of various 650 artificial neural networks for monthly streamflow forecasting. Water, 10(5), 628.

70. Zhang, X., Zhang, Q., Zhang, G., Nie, Z., Gui, Z., \& Que, H. (2018). A novel hybrid datadriven model for daily land surface temperature forecasting using long short-term memory 
652 neural network based on ensemble empirical mode decomposition. International journal of 653 environmental research and public health, 15(5), 1032.

654 71. Zheng, Y. J., Chen, S. Y., Lin, Y., \& Wang, W. L. (2013). Bio-inspired optimization of 655 sustainable energy systems: a review. Mathematical Problems in Engineering, 2013. 
Figure 1

Figure 1

The schematic view of proposed model i.e. SSA-VMD-EBT-SVM model.

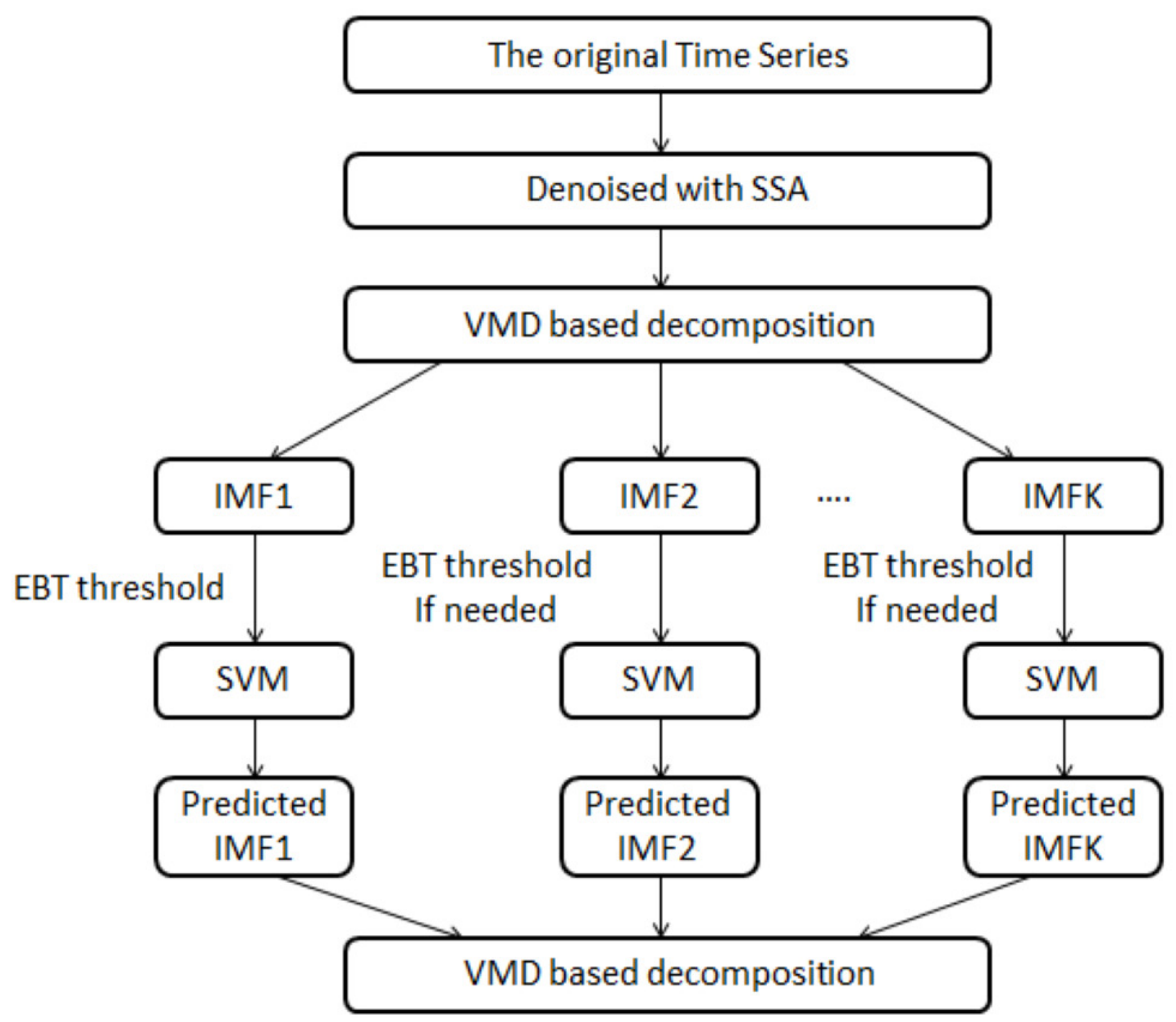


Figure 2

Figure 2

Figure 2 : Eigenvalues of SSA components. (A) Indus river inflow (B) Jhelum river inflow
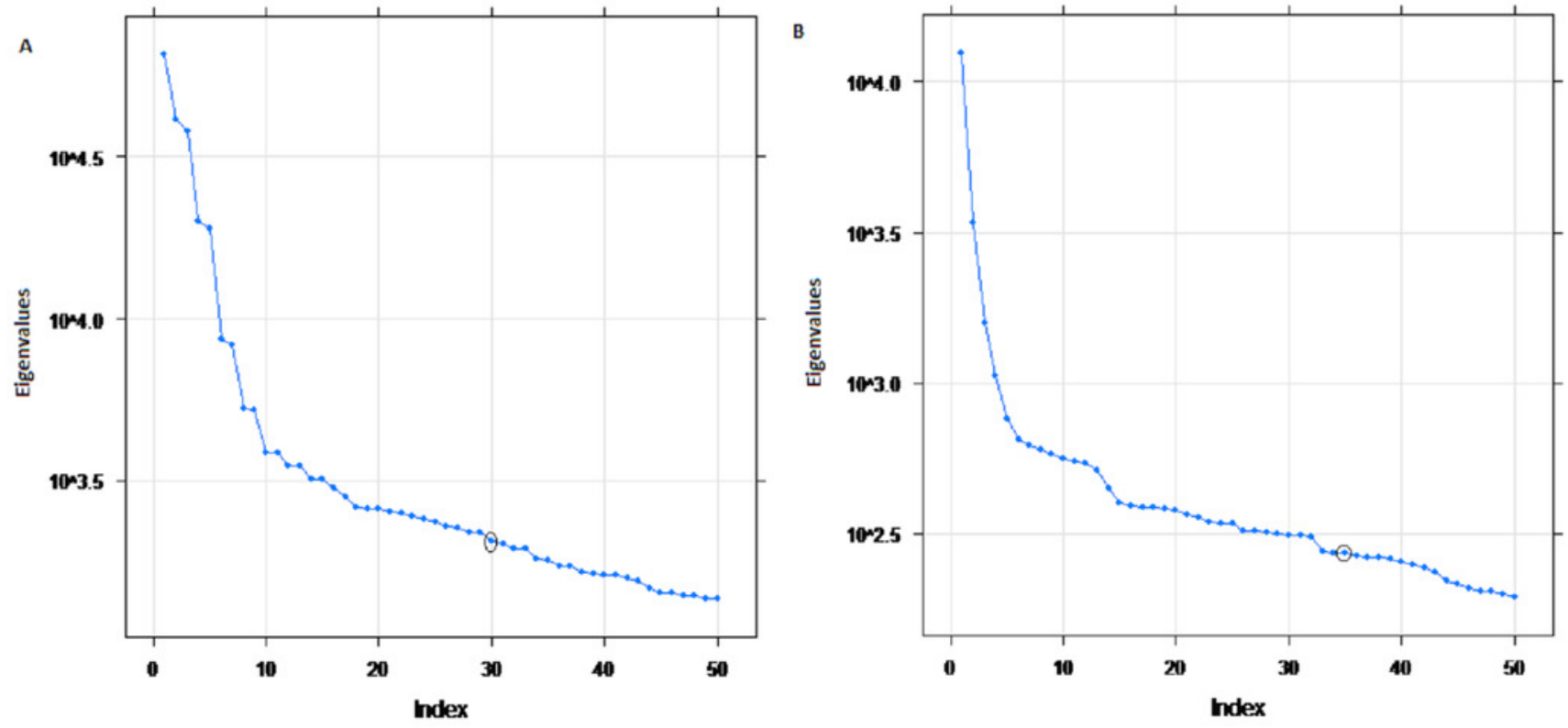
Figure 3

Figure 3

Figure 3: Processed inflow data through SSA. (A) For Indus river inflow (B) For Jhelum river inflow 

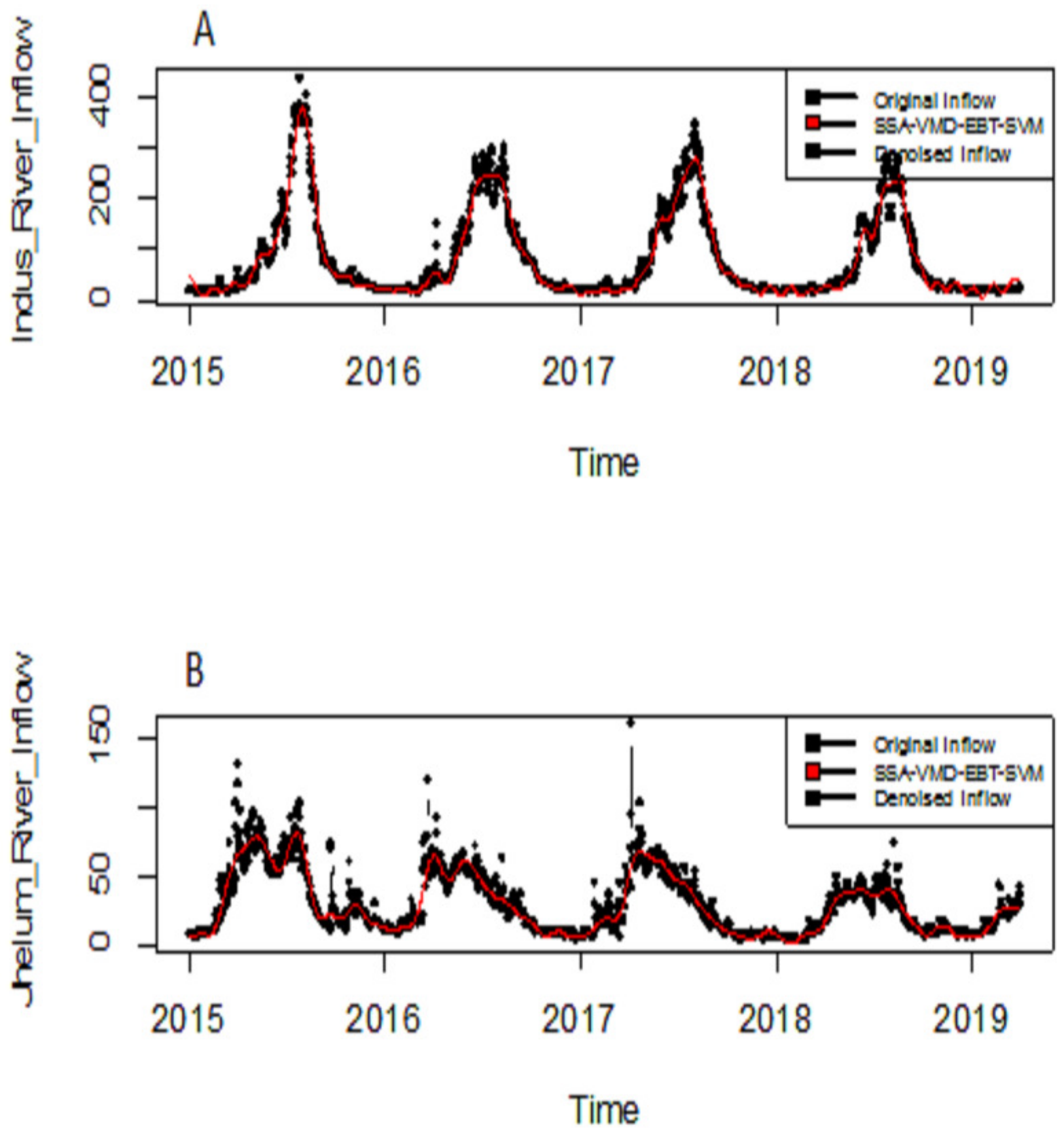
Figure 4

Figure 4

Figure 4: The SSA-VMD based decomposition of Indus river inflow. The series is decomposed into five IMFs and one residue. 


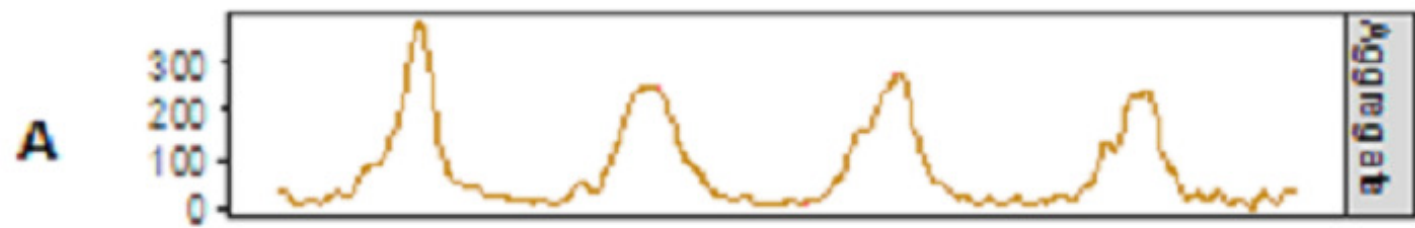

B $\quad \begin{array}{r}80 \\ 70 \\ 60\end{array}$
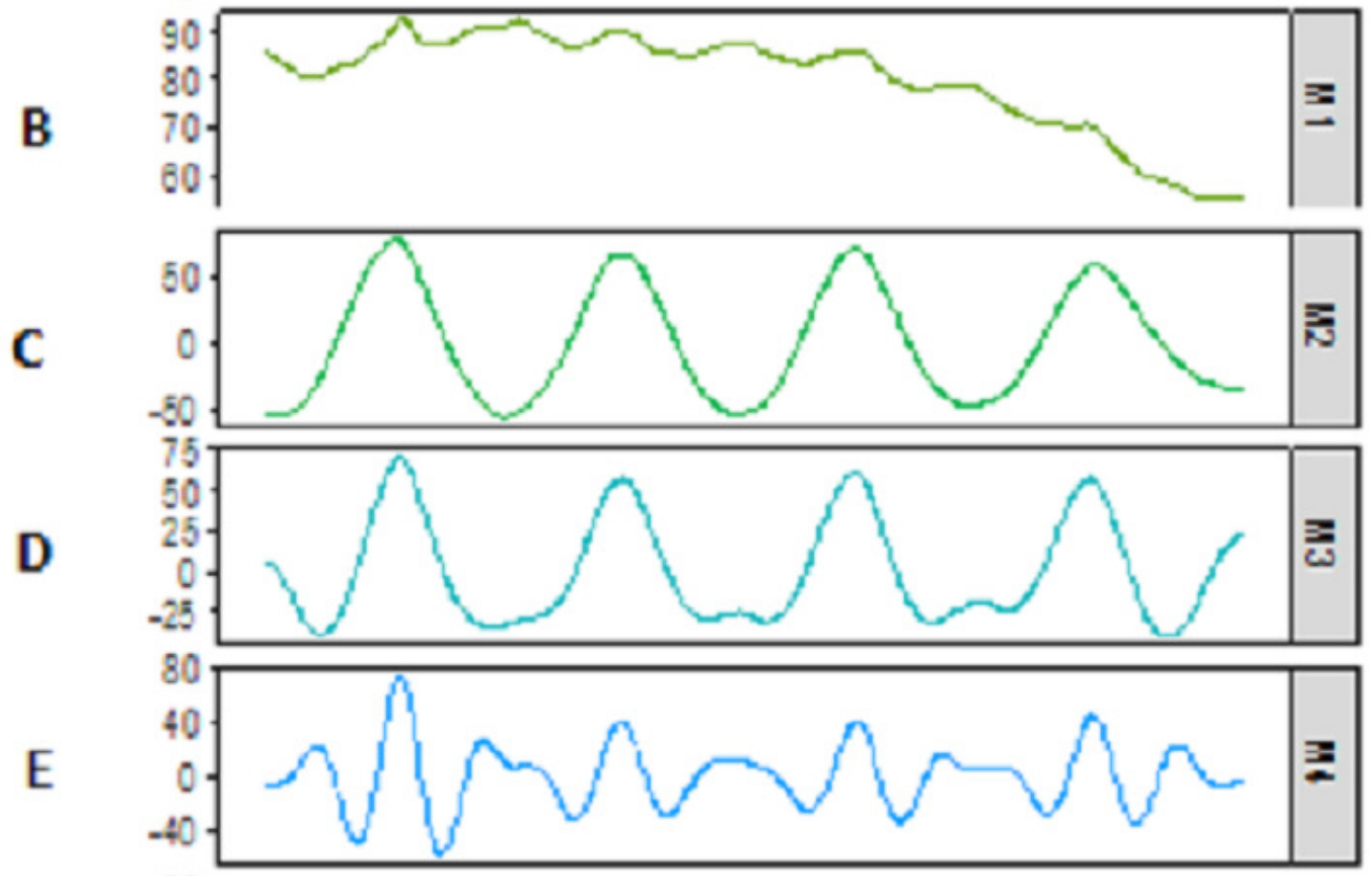

F

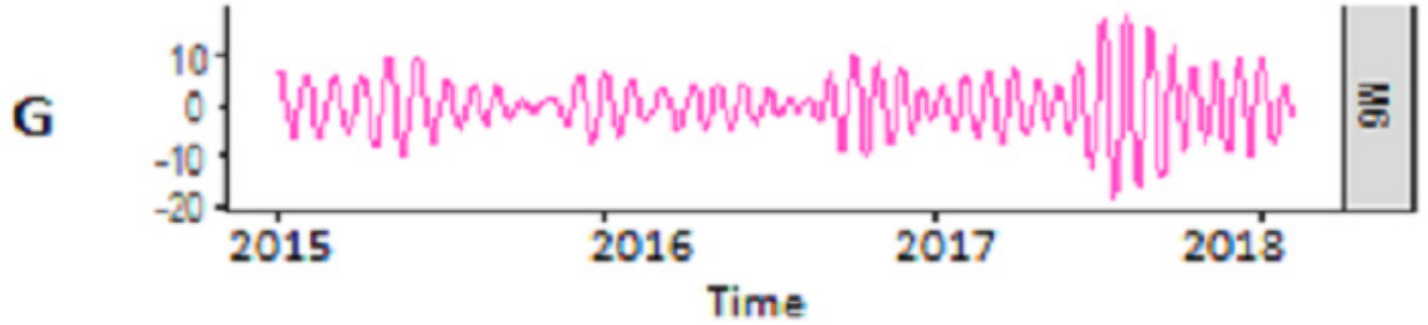


Figure 5

Figure 5

Figure 5: The SSA-EMD based decomposition of Indus river inflow. The series is decomposed into five IMFs and one residue.

A

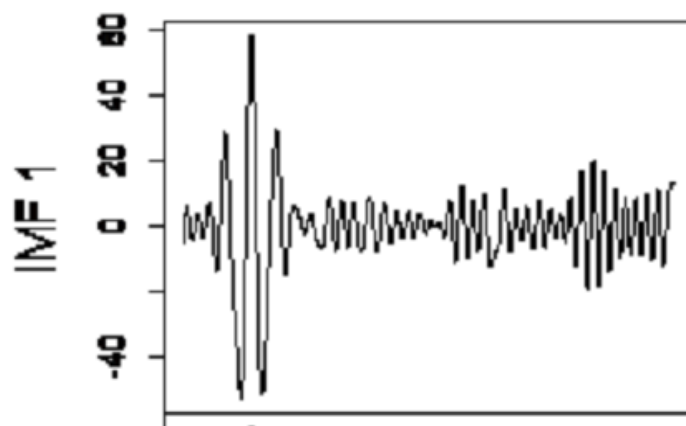

B
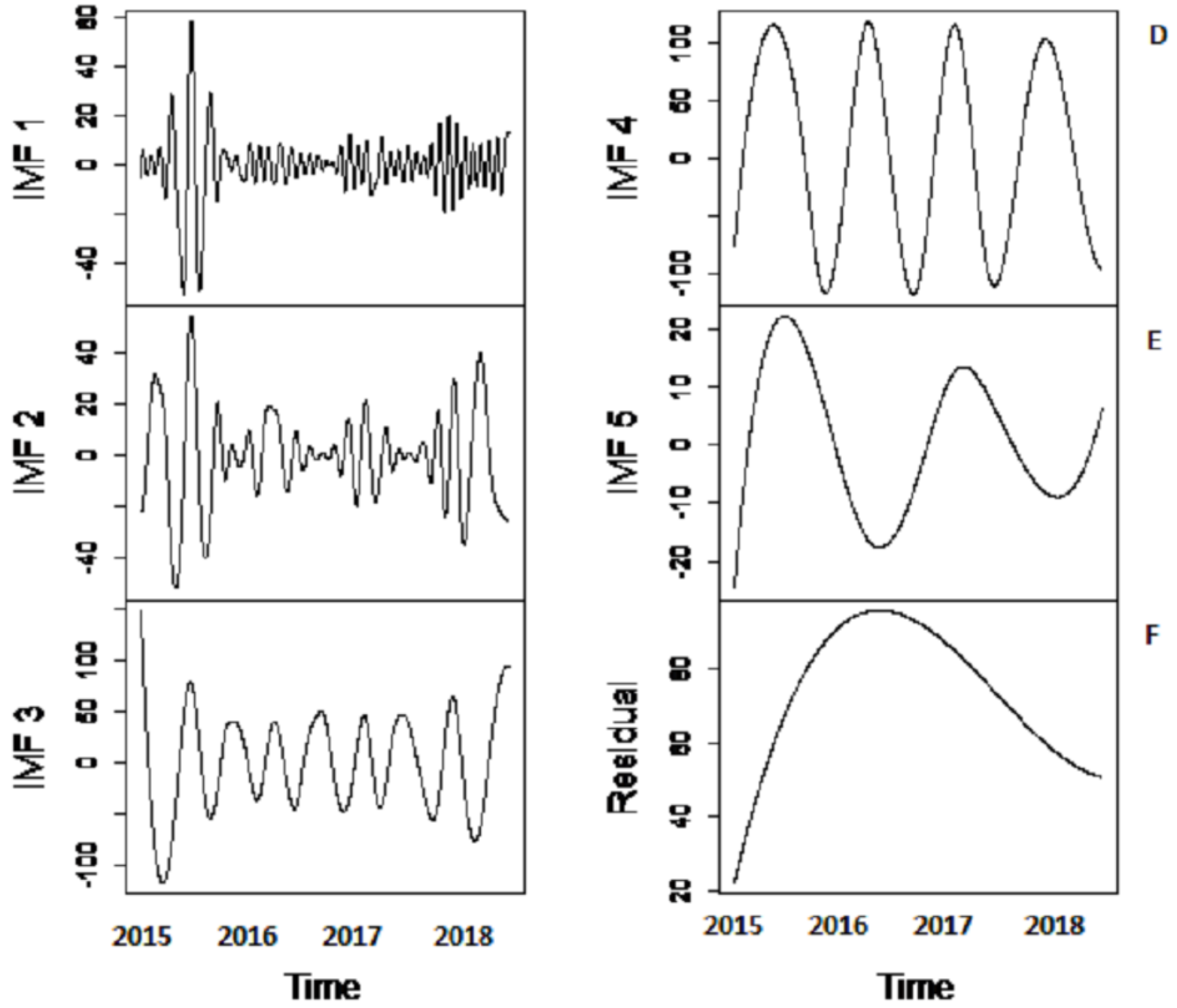
Figure 6

Figure 6

Figure 6: The SSA-EEMD based decomposition of Indus river inflow. The series is decomposed into five IMFs and one residue

A

B

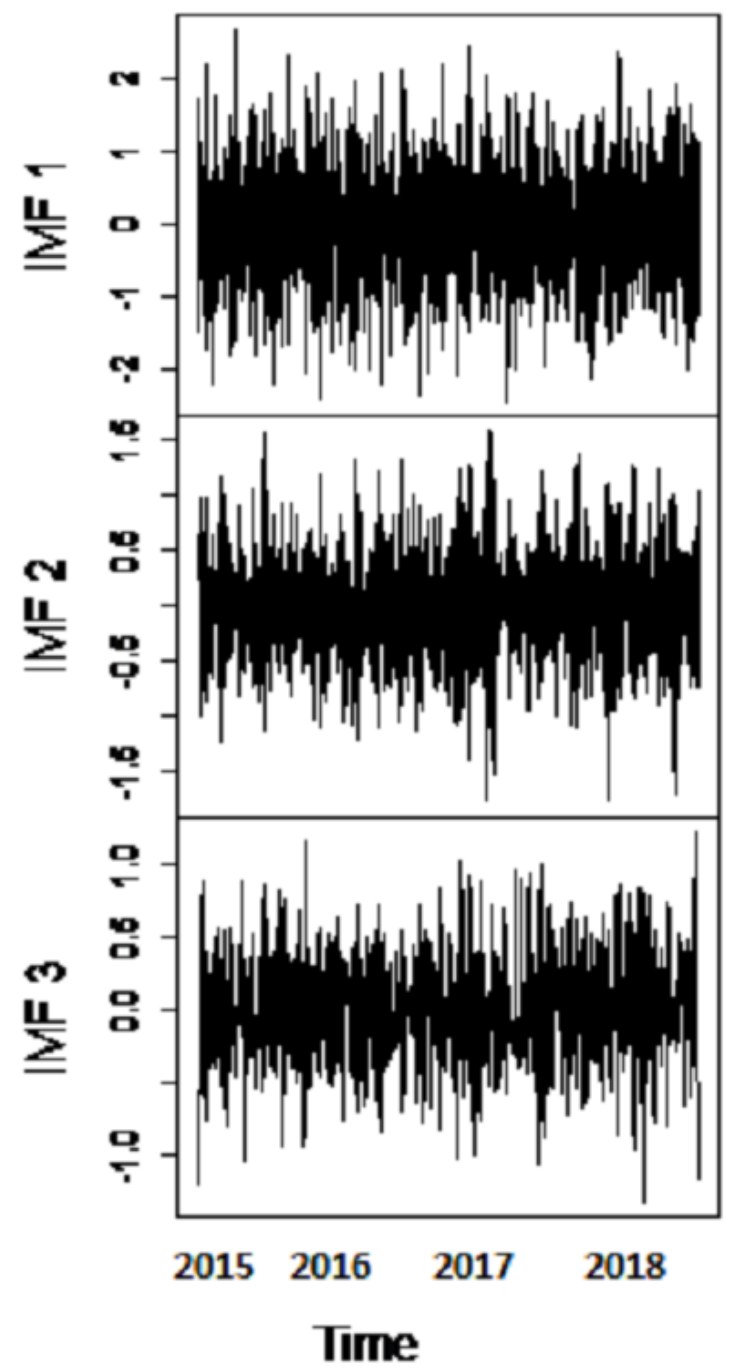

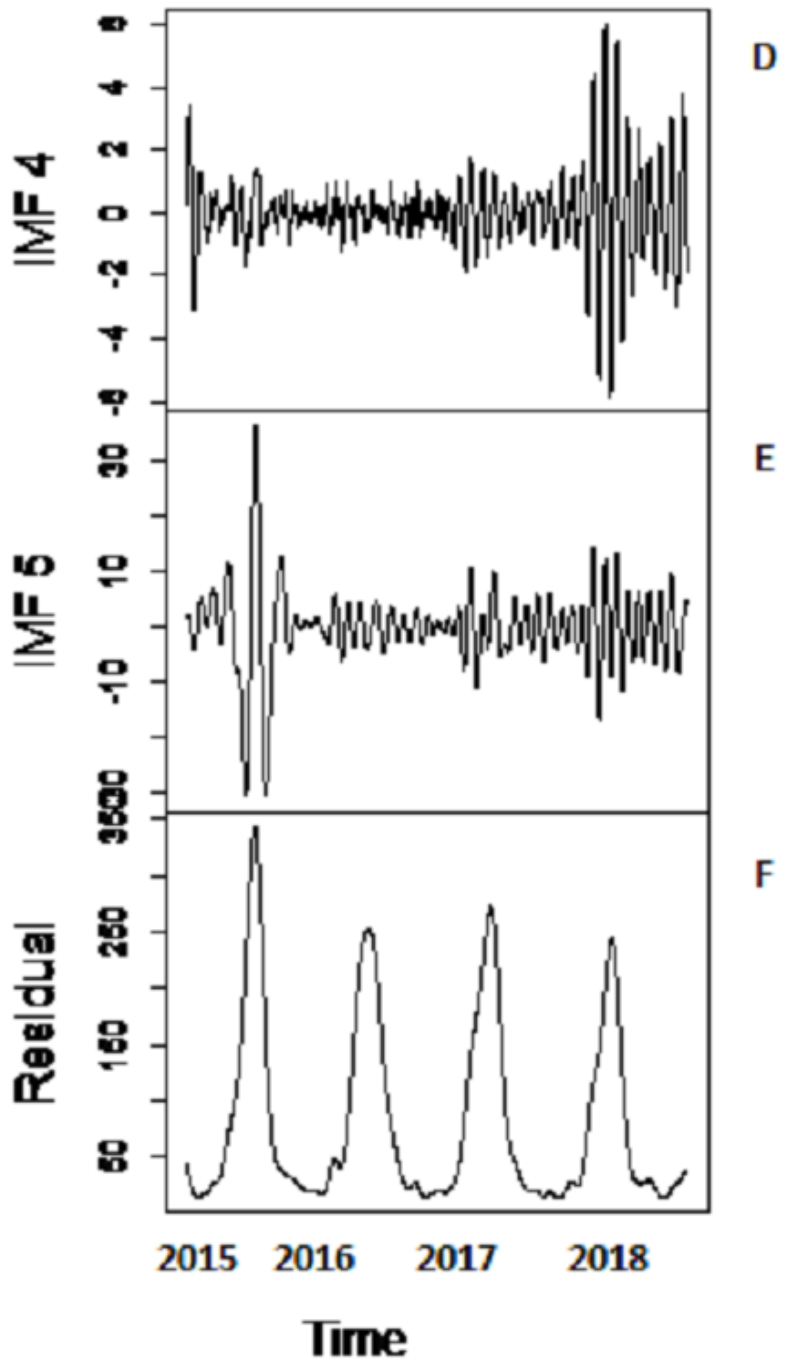




\section{Figure 7}

Figure 7

Figure 7: Taylor diagram: performance measures for Indus (Left) and Jhelum (Right) river inflow 
A

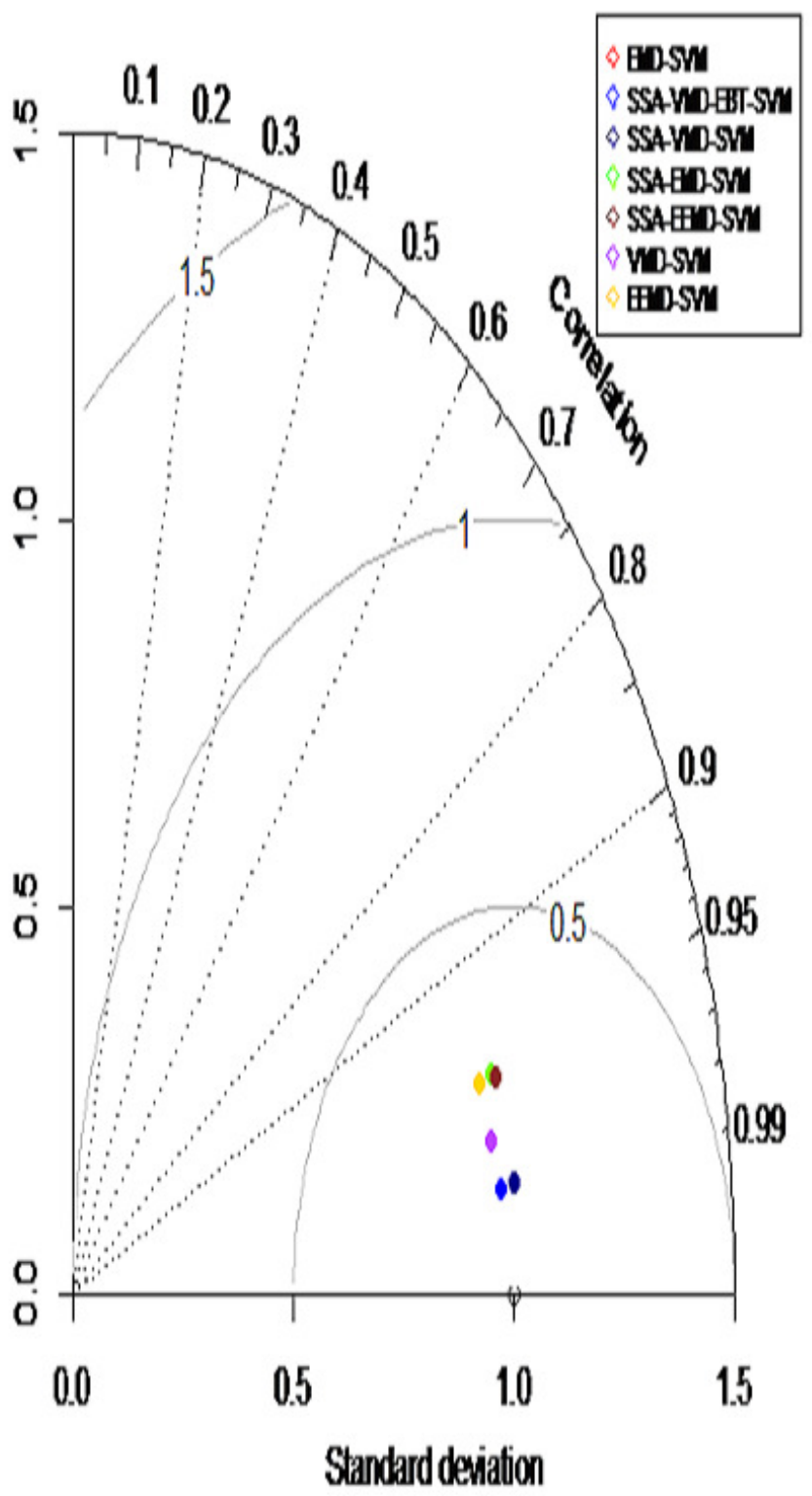

B

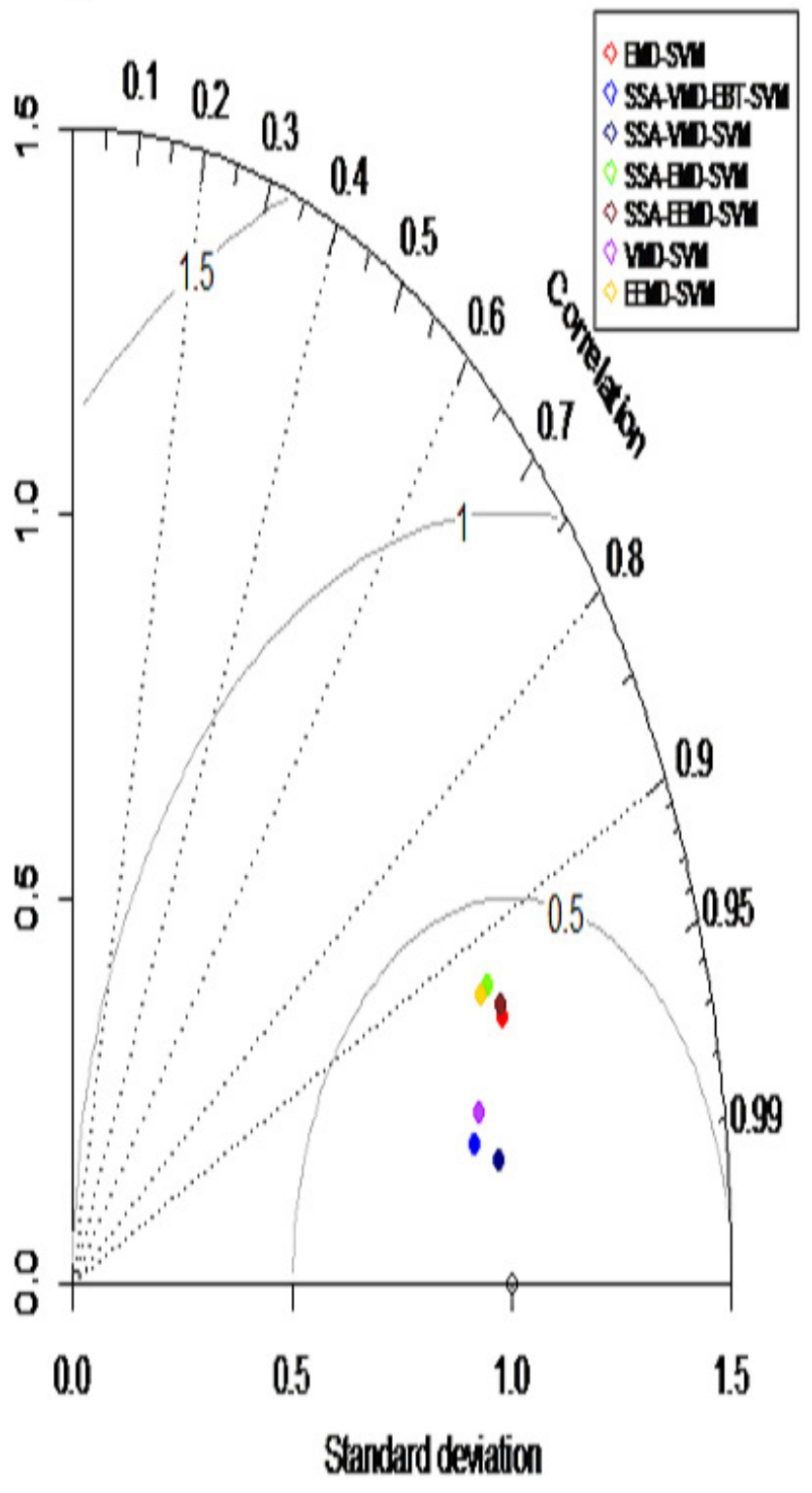


Figure 8

Figure 8

Figure 8: Validation graph of proposed SSA-VMD-EBT-MM and all other benchmark models. (A) For Indus river inflow (B) For Jhelum river inflow. 

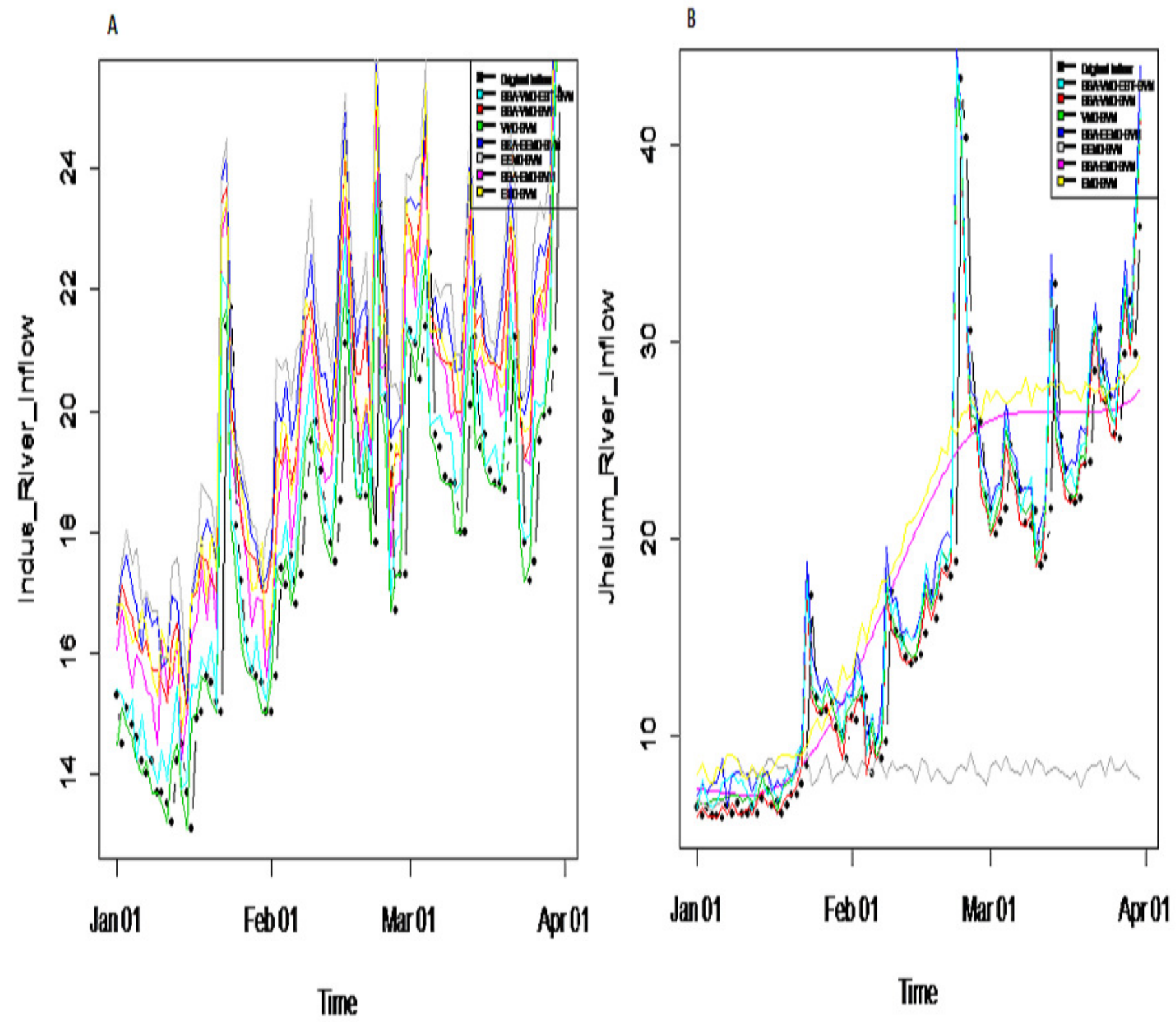


\section{Table 1 (on next page)}

Test statistics and critical values of the ADF test for all four rivers of IRB system. 


\begin{tabular}{c|c|c}
\hline River inflow & Test Statistic & Critical Values \\
\hline Indus river & -2.8482 & 0.2192 \\
Jhelum river & -2.9841 & 0.1617 \\
Chenab river & -3.2363 & 0.0817 \\
Kabul river & -2.8369 & 0.2240
\end{tabular}

1 Table 1: Test statistics and critical values of the ADF test for all four rivers of IRB system. 2 


\section{Table 2 (on next page)}

Summary statistics of original and denoised rivers inflow data.

SD* shows standard deviation value. 
1

\begin{tabular}{ccccc}
\hline \multirow{2}{*}{ River inflow } & \multicolumn{2}{c}{ Original inflow } & \multicolumn{2}{c}{ Denoised inflow } \\
\cline { 2 - 5 } & Mean & SD* & Mean & SD* \\
\hline Indus river & 79.0805 & 86.1221 & 79.9920 & 84.0473 \\
Jhelum river & 28.0692 & 22.5040 & 28.1654 & 21.2862 \\
Chenab river & 27.3528 & 24.6649 & 27.1066 & 23.4877 \\
Kabul river & 31.7901 & 29.4850 & 31.4863 & 27.6831
\end{tabular}

2 Table 2: Summary statistics of original and denoised rivers inflow data. SD* shows standard 3 deviation value.

4 


\section{Table 3 (on next page)}

Evaluation index of the training prediction error of the proposed model (SSA-VMD-EBT$M M)$ with all selected models for Indus, Jhelum and Chenab river inflow. NSE and MAPE are unit free measures whenever, MSE, RMSE and MAE are measure in $1000 \mathrm{ft} / \mathrm{s}$. 


\begin{tabular}{|c|c|c|c|c|c|c|}
\hline Station & Models & NSE & MSE & RMSE & MAE & MAPE \\
\hline \multirow{7}{*}{ Tarbela } & EMD-SVM & 0.9946 & 41.2868 & 6.4255 & 5.8657 & 16.9818 \\
\hline & EEMD-SVM & 0.9962 & 39.3084 & 5.4137 & 4.5540 & 11.3814 \\
\hline & SSA-EMD-SVM & 0.9953 & 34.0684 & 5.8368 & 4.9262 & 12.9763 \\
\hline & SSA-EEMD-SVM & 0.9962 & 28.0560 & 5.9551 & 4.9610 & 12.2437 \\
\hline & VMD-SVM & 0.9911 & 68.2308 & 8.2602 & 6.8929 & 19.5466 \\
\hline & SSA-VMD-SVM & 0.9954 & 33.5565 & 5.7928 & 5.2533 & 15.60666 \\
\hline & SSA-VMD-EBT-SVM & 0.9965 & 32.7355 & 5.7215 & 4.7800 & 10.9320 \\
\hline \multirow{7}{*}{ Mangla } & EMD-SVM & 0.9396 & 31.6725 & 5.6278 & 3.2197 & 16.5250 \\
\hline & EEMD-SVM & 0.9753 & 12.9495 & 3.5985 & 2.2437 & 12.1502 \\
\hline & SSA-EMD-SVM & 0.9912 & 4.1259 & 2.0312 & 1.8052 & 17.0294 \\
\hline & SSA-EEMD-SVM & 0.9769 & 10.8790 & 3.2984 & 2.4393 & 17.4766 \\
\hline & VMD-SVM & 0.9712 & 15.1061 & 3.8866 & 2.4723 & 14.8790 \\
\hline & SSA-VMD-SVM & 0.9731 & 12.6437 & 3.5558 & 2.7425 & 21.2450 \\
\hline & SSA-VMD-EBT-SVM & 0.9936 & 3.0168 & 1.7369 & 1.7979 & 7.9978 \\
\hline \multirow{7}{*}{ Marala } & EMD-SVM & 0.9760 & 14.9508 & 3.8666 & 2.1770 & 10.9986 \\
\hline & EEMD-SVM & 0.9893 & 6.6943 & 2.5873 & 2.7507 & 10.8725 \\
\hline & SSA-EMD-SVM & 0.9748 & 14.1228 & 3.7581 & 2.8346 & 48.3824 \\
\hline & SSA-EEMD-SVM & 0.9796 & 11.4645 & 3.3859 & 2.5789 & 28.3756 \\
\hline & VMD-SVM & 0.6525 & 216.738 & 14.72202 & 9.9701 & 47.295 \\
\hline & SSA-VMD-SVM & 0.9780 & 12.3627 & 3.5161 & 2.6684 & 38.8350 \\
\hline & SSA-VMD-EBT-SVM & 0.9953 & 6.3790 & 2.5257 & 2.0556 & 10.8084 \\
\hline
\end{tabular}

1 Table 3: Evaluation index of the training prediction error of the proposed model (SSA-VMD-EBT-

2 MM) with all selected models for Indus, Jhelum and Chenab river inflow. NSE and MAPE are

3 unit free measures whenever, MSE, RMSE and MAE are measure in $1000 \mathrm{ft} / \mathrm{s}$. 


\section{Table 4 (on next page)}

Evaluation index of the training prediction error of the proposed model (SSA-VMD-EBTMM) with all selected models for Kabul river inflow. NSE and MAPE are unit free measures whenever, MSE, RMSE and MAE are measure in $1000 \mathrm{ft} / \mathrm{s}$. 


\begin{tabular}{lcccccc}
\hline Station & Models & NSE & MSE & RMSE & MAE & MAPE \\
\hline \multirow{3}{*}{ Nowshera } & EMD-SVM & 0.9156 & 75.6570 & 8.6981 & 4.5491 & 19.2872 \\
& EEMD-SVM & 0.9639 & 32.3744 & 5.6898 & 3.0101 & 12.2574 \\
& SSA-EMD-SVM & 0.9820 & 14.1973 & 3.7679 & 2.9610 & 38.3520 \\
& VMD-SVM & 0.9585 & 37.2792 & 6.1057 & 3.4760 & 17.8121 \\
& SSA-VMD-SVM & 0.9946 & 4.2555 & 2.0629 & 1.8699 & 21.0699 \\
& SSA-VMD-EBT-SVM & $\mathbf{0 . 9 9 4 5}$ & $\mathbf{4 . 2 5 5 5}$ & $\mathbf{2 . 0 6 2 8}$ & $\mathbf{1 . 8 6 9 9}$ & $\mathbf{2 1 . 0 6 9 9}$
\end{tabular}

1 Table 4: Evaluation index of the training prediction error of the proposed model (SSA-VMD-

2 EBT-MM) with all selected models for Kabul river inflow. NSE and MAPE are unit free

3 measures whenever, MSE, RMSE and MAE are measure in $1000 \mathrm{ft} / \mathrm{s}$. 